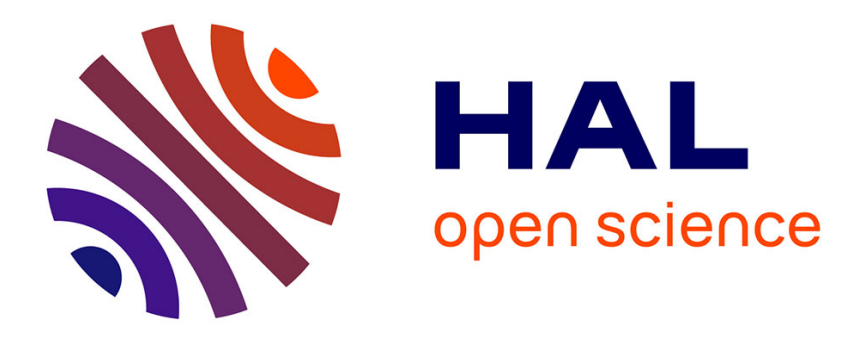

\title{
Corrélation entre les amplifications géniques et l'imagerie TEP à la 18F-DOPA dans les glioblastomes récidivants
}

Romain Capet

\section{- To cite this version:}

Romain Capet. Corrélation entre les amplifications géniques et l'imagerie TEP à la 18F-DOPA dans les glioblastomes récidivants. Médecine humaine et pathologie. 2017. dumas-01985171

\section{HAL Id: dumas-01985171 https://dumas.ccsd.cnrs.fr/dumas-01985171}

Submitted on 17 Jan 2019

HAL is a multi-disciplinary open access archive for the deposit and dissemination of scientific research documents, whether they are published or not. The documents may come from teaching and research institutions in France or abroad, or from public or private research centers.
L'archive ouverte pluridisciplinaire $\mathbf{H A L}$, est destinée au dépôt et à la diffusion de documents scientifiques de niveau recherche, publiés ou non, émanant des établissements d'enseignement et de recherche français ou étrangers, des laboratoires publics ou privés. 
UNIVERSITÉ DE NICE SOPHIA ANTIPOLIS

FACULTÉ DE MÉDECINE

\section{THÈSE D'EXERCICE DE MÉDECINE}

DIPLOME D’ÉTAT DE DOCTEUR EN MÉDECINE

Corrélation entre les amplifications géniques et l'imagerie TEP à la 18F-DOPA dans les glioblastomes récidivants

Présentée et soutenue publiquement le 2 novembre 2017

Par

Romain CAPET

Né le 14 février 1988 à Pau (64)

Discipline : médecine nucléaire

Président du jury

M. le Professeur J. DARCOURT

Directrice de thèse

Mme. Le Docteur F. BUREL-VANDENBOS

Assesseurs

M. le Professeur P. PAQUIS

Mme le Professeur F. PEDEUTOUR 


\section{UNIVERSITÉ NICE-SOPHIA ANTIPOLIS \\ FACULTÉ DE MÉDECINE

Liste des professeurs au 1er septembre 2017 à la Faculté de Médecine de Nice

\section{Doyen}

Vice-Doyen

Assesseurs

Conservateur de la bibliothèque

Directrice administrative des services

Doyens Honoraires

\section{BAQUÉ Patrick}

M. BOILEAU Pascal

M. ESNAULT Vincent $M$ DELLAMONICA Jean Mme BREUIL Véronique M. MARTY Pierre

Mme AMSELLE Danièle

Mme CALLEA Isabelle

M. AYRAUD Noël

M. RAMPAL Patrick

M. BENCHIMOL Daniel

Professeurs Honoraires

M ALBERTINI Marc

M. BALAS Daniel

M. BATT Michel

M. BLAIVE Bruno

M. BOQUET Patrice

M. BOURGEON André

M. BOUTTÉ Patrick

M. BRUNETON Jean-Noël

Mme BUSSIERE Françoise

M. CAMOUS Jean-Pierre

M. CANIVET Bertrand

M. CASSUTO Jill-patrice

M. CHATEL Marcel

M. COUSSEMENT Alain Mme CRENESSE Dominique

M. DARCOURT GUY

M. DELLAMONICA Pierre

M. DELMONT Jean

M. DEMARD François

M. DESNUELLE Claude

M. DOLISI Claude

$M$. FRANCO Alain

M. FREYCHET Pierre

M. GÉRARD Jean-Pierre

M. GILLET Jean-Yves
M. GRELLIER Patrick

M. GRIMAUD Dominique

$M$. HARTER Michel

M. INGLESAKIS Jean-André

M. JOURDAN Jacques

M. LALANNE Claude-Michel

M. LAMBERT Jean-Claude

M. LAZDUNSKI Michel

M. LEFEBVRE Jean-Claude

M. LE FICHOUX Yves Mme LEBRETON Elisabeth

M. LOUBIERE Robert

M. MARIANI Roger

M. MASSEYEFF René

M. MATTEI Mathieu

M. MOUIEL Jean

Mme MYQUEL Martine

M. ORTONNE Jean-Paul

M. PRINGUEY Dominique

M. SAUTRON Jean Baptiste

M. SCHNEIDER Maurice

M. TOUBOL Jacques

M. TRAN Dinh Khiem

$M$ VAN OBBERGHEN Emmanuel

M. ZIEGLER Gérard 
UNIVERSITÉ NICE-SOPHIA ANTIPOLIS

FACULTÉ DE MÉDECINE

\section{Faculté de Médecine}

Liste des professeurs au 1er septembre 2017 à la Faculté de Médecine de Nice

M.C.A. Honoraire

M.C.U. Honoraires
Mlle ALLINE Madeleine
M. ARNOLD Jacques
M. BASTERIS Bernard
MIle CHICHMANIAN Rose-Marie
Mme DONZEAU Michèle
M. EMILIOZZI Roméo
M. FRANKEN Philippe
M. GASTAUD Marcel
M. GIUDICELLI Jean
M. MAGNÉ Jacques
Mme MEMRAN Nadine
M. MENGUAL Raymond
M. PHILIP Patrick
M. POIRÉE Jean-Claude
Mme ROURE Marie-Claire 
UNIVERSITÉ NICE-SOPHIA ANTIPOLIS

FACULTÉ DE MÉDECINE

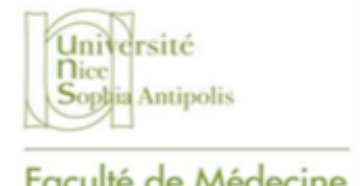

Faculté de Médecine

Liste des professeurs au 1er septembre 2017 à la Faculté de Médecine de Nice

\section{PROFESSEURS CLASSE EXCEPTIONNELLE}

$\begin{array}{ll}\text { M. } & \text { AMIEL Jean } \\ \text { M. } & \text { BERNARDIN Gilles } \\ \text { M. } & \text { BOILEAU Pascal } \\ \text { M. } & \text { DARCOURT Jacques } \\ \text { M. } & \text { ESNAULT Vincent } \\ \text { Mme } & \text { EULLER-ZIEGLER Liana } \\ \text { M. } & \text { FENICHEL Patrick } \\ \text { M. } & \text { FUZIBET Jean-Gabriel } \\ \text { M. } & \text { GASTAUD Pierre } \\ \text { M. } & \text { GILSON Éric } \\ \text { M. } & \text { HASSEN KHODJA Reda } \\ \text { M. } & \text { HÉBUTERNE Xavier } \\ \text { M. } & \text { HOFMAN Paul } \\ \text { Mme } & \text { ICHAI Carole } \\ \text { M. } & \text { LACOUR Jean-Philippe } \\ \text { M. } & \text { LEFTHERIOTIS Geogres } \\ \text { M. } & \text { MARQUETTE Charles-Hugo } \\ \text { M. } & \text { MARTY Pierre } \\ \text { M. } & \text { MICHIELS Jean-François } \\ \text { M. } & \text { MOUROUX Jérôme } \\ \text { Mme } & \text { PAQUIS Véronique } \\ \text { M. } & \text { PAQUIS Philippe } \\ \text { M. } & \text { QUATREHOMME Gérald } \\ \text { M. } & \text { RAUCOULES-AIMÉ Marc } \\ \text { M. } & \text { ROBERT Philippe } \\ \text { M. } & \text { SANTINI Joseph } \\ \text { M. } & \text { THYSS Antoine } \\ \text { M. } & \text { TRAN Albert } \\ & \end{array}$

Urologie (52.04)

Réanimation Médicale (48.02)

Chirurgie Orthopédique et Traumatologique (50.02)

Biophysique et Médecine Nucléaire (43.01)

Néphrologie (52-03)

Rhumatologie (50.01)

Biologie du Développement et de la Reproduction (54.05)

Médecine Interne (53.01)

Ophtalmologie (55.02)

Biologie Cellulaire (44.03)

Chirurgie Vasculaire (51.04)

Nutrition (44.04)

Anatomie et Cytologie Pathologiques (42.03)

Anesthésiologie et Réanimation Chirurgicale (48.01)

Dermato-Vénéréologie (50.03)

Physiologie- médecine vasculaire

Pneumologie (51.01)

Parasitologie et Mycologie (45.02)

Anatomie et Cytologie Pathologiques (42.03)

Chirurgie Thoracique et Cardiovasculaire (51.03)

Génétique (47.04)

Neurochirurgie (49.02)

Médecine Légale et Droit de la Santé (46.03)

Anesthésie et Réanimation Chirurgicale (48.01)

Psychiatrie d'Adultes (49.03)

O.R.L. (55.01)

Cancérologie, Radiothérapie (47.02)

Hépato Gastro-entérologie (52.01) 
UNIVERSITÉ NICE-SOPHIA ANTIPOLIS

FACULTÉ DE MÉDECINE

Faculté de Médecine

Liste des professeurs au 1er septembre 2017 à la Faculté de Médecine de Nice

\section{PROFESSEURS PREMIERE CLASSE}

$\begin{array}{ll}\text { Mme } & \text { ASKENAZY-GITTARD Florence } \\ \text { M. } & \text { BAQUÉ Patrick } \\ \text { M. } & \text { BARRANGER Emmanuel } \\ \text { M. } & \text { BÉRARD Étienne } \\ \text { Mme } & \text { BLANC-PEDEUTOUR Florence } \\ \text { M. } & \text { BONGAIN André } \\ \text { Mme } & \text { BREUIL Véronique } \\ \text { M. } & \text { CASTILLO Laurent } \\ \text { M. } & \text { DE PERETTI Fernand } \\ \text { M. } & \text { DRICI Milou-Daniel } \\ \text { M. } & \text { FERRARI Émile } \\ \text { M. } & \text { FERRERO Jean-Marc } \\ \text { M. } & \text { GIBELIN Pierre } \\ \text { M. } & \text { GUGENHEIM Jean } \\ \text { M. } & \text { HANNOUN-LEVI Jean-Michel } \\ \text { M. } & \text { LONJON Michel } \\ \text { M. } & \text { MOUNIER Nicolas } \\ \text { M. } & \text { PADOVANI Bernard } \\ \text { M. } & \text { PICHE Thierry } \\ \text { M. } & \text { PRADIER Christian } \\ \text { Mme } & \text { RAYNAUD Dominique } \\ \text { M. } & \text { ROSENTHAL Éric } \\ \text { M. } & \text { SCHNEIDER Stéphane } \\ \text { M. } & \text { STACCINI Pascal } \\ \text { M. } & \text { THOMAS Pierre } \\ & \end{array}$

Pédopsychiatrie (49.04)

Anatomie - Chirurgie Générale (42.01)

Gynécologie Obstétrique (54.03)

Pédiatrie (54.01)

Cancérologie - Génétique (47.02)

Gynécologie-Obstétrique (54.03)

Rhumatologie (50.01)

O.R.L. (55.01)

Anatomie-Chirurgie Orthopédique (42.01)

Pharmacologie Clinique (48.03)

Cardiologie (51.02)

Cancérologie ; Radiothérapie (47.02)

Cardiologie (51.02)

Chirurgie Digestive (52.02)

Cancérologie ; Radiothérapie (47.02)

Neurochirurgie (49.02)

Cancérologie, Radiothérapie (47.02)

Radiologie et Imagerie Médicale (43.02)

Gastro-entérologie (52.01)

Épidémiologie, Économie de la Santé et Prévention (46.01)

Hématologie (47.01)

Médecine Interne (53.01)

Nutrition (44.04)

Biostatistiques et Informatique Médicale (46.04)

Neurologie (49.01) 
UNIVERSITÉ NICE-SOPHIA ANTIPOLIS

FACULTÉ DE MÉDECINE

\section{Faculté de Médecine}

Liste des professeurs au 1er septembre 2017 à la Faculté de Médecine de Nice

\section{PROFESSEURS DEUXIEME CLASSE}

Mme ALUNNI Véronique

M. ANTY Rodolphe

M. BAHADORAN Philippe

Mme BAILLIF Stéphanie

M. BENIZRI Emmanuel

M. BENOIT Michel

M. BREAUD Jean

M. CARLES Michel

M. CHEVALIER Nicolas

M. CHEVALLIER Patrick

Mme CHINETTI Giulia

M. CLUZEAU Thomas

M. DELLAMONICA Jean

M. DELOTTE Jérôme

M. FONTAINE Denys

M. FOURNIER Jean-Paul

Mlle GIORDANENGO Valérie

M. GUÉRIN Olivier

M. IANNELLI Antonio

$M \quad J E A N$ BAPTISTE Elixène

M. LEVRAUT Jacques

M. PASSERON Thierry

M. ROGER Pierre-Marie

M. ROHRLICH Pierre

M. ROUX Christian

M. RUIMY Raymond

Mme SACCONI Sabrina

M. SADOUL Jean-Louis

M. TROJANI Christophe

M. VENISSAC Nicolas
Médecine Légale et Droit de la Santé (46.03)

Gastro-entérologie (52.01)

Cytologie et Histologie (42.02)

Ophtalmologie (55.02)

Chirurgie Générale (53.02)

Psychiatrie (49.03)

Chirurgie Infantile (54-02)

Anesthésiologie Réanimation (48.01)

Endocrinologie, Diabète et Maladies Métaboliques (54.04)

Radiologie et Imagerie Médicale (43.02)

Biochimie-Biologie Moléculaire (44.01)

Hématologie (47.01)

réanimation médicale (48.02)

Gynécologie-obstétrique (54.03)

Neurochirurgie (49.02)

Thérapeutique (48-04)

Bactériologie-Virologie (45.01)

Gériatrie (48.04)

Chirurgie Digestive (52.02)

Chirurgie vasculaire (51.04)

Anesthésiologie et Réanimation Chirurgicale (48.01)

Dermato-Vénéréologie (50-03)

Maladies Infectieuses; Maladies Tropicales (45.03)

Pédiatrie (54.01)

rhumatologie (50.01)

Bactériologie-virologie (45.01)

Neurologie (49.01)

Endocrinologie, Diabète et Maladies Métaboliques (54.04)

Chirurgie Orthopédique et Traumatologique (50.02)

Chirurgie Thoracique et Cardiovasculaire (51.03) 


\section{UNIVERSITÉ NICE-SOPHIA ANTIPOLIS \\ FACULTÉ DE MÉDECINE

\section{Liste des professeurs au 1er septembre 2017 à la Faculté de Médecine de Nice}

\section{PROFESSEUR DES UNIVERSITÉS}

M. HOFLIGER Philippe Médecine Générale (53.03)

\section{MAITRE DE CONFÉRENCES DES UNIVERSITÉS}

M. DARMON David

Médecine Générale (53.03)

PROFESSEURS AGRÉGÉS

Mme LANDI Rebecca Anglais

\section{MAITRES DE CONFÉRENCES DES UNIVERSITÉS - PRATICIENS HOSPITALIERS}

\begin{tabular}{ll} 
M. & AMBROSETTI Damien \\
Mme & BANNWARTH Sylvie \\
M. & BENOLIEL José \\
Mme & BERNARD-POMIER Ghislaine \\
M. & BRONSARD Nicolas \\
Mme & BUREL-VANDENBOS Fanny \\
M. & DOGLIO Alain \\
M & DOYEN Jérôme \\
M & FAVRE Guillaume \\
M. & FOSSE Thierry \\
M. & GARRAFFO Rodolphe \\
Mme & GIOVANNINI-CHAMI Lisa \\
Mme & HINAULT Charlotte \\
M. & HUMBERT Olivier \\
Mme & LAMY Brigitte \\
Mme & LEGROS Laurence \\
Mme & LONG-MIRA Elodie \\
Mme & MAGNIÉ Marie-Noëlle \\
Mme & MOCERI Pamela \\
Mme & MUSSO-LASSALLE Sandra \\
M. & NAÏMI Mourad \\
Mme & POMARES Christelle \\
Mme & SEITZ-POLSKI barbara \\
M. & TESTA Jean \\
M. & TOULON Pierre \\
& \\
\hline
\end{tabular}

Cytologie et Histologie (42.02)

Génétique (47.04)

Biophysique et Médecine Nucléaire (43.01)

Immunologie (47.03)

Anatomie Chirurgie Orthopédique et Traumatologique (42.01)

Anatomie et Cytologie pathologiques (42.03)

Bactériologie-Virologie (45.01)

Radiothérapie (47.02)

Néphrologie (52.03)

Bactériologie-Virologie-Hygiène (45.01)

Pharmacologie Fondamentale (48.03)

Pédiatrie (54.01)

Biochimie et biologie moléculaire (44.01)

Biophysique et Médecine Nucléaire (43.01)

Bactérilogie-virologie ( 45.01)

Hématologie et Transfusion (47.01)

Cytologie et Histologie (42.02)

Physiologie (44.02)

Cardiologie (51.02)

Anatomie et Cytologie pathologiques (42.03)

Biochimie et Biologie moléculaire (44.01)

Parasitologie et mycologie (45.02)

Immunologie (47.03)

Épidémiologie Économie de la Santé et Prévention (46.01)

Hématologie et Transfusion (47.01) 


\section{UNIVERSITÉ NICE-SOPHIA ANTIPOLIS \\ FACULTÉ DE MÉDECINE}

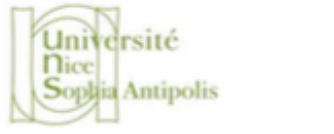

Faculté de Médecine

Liste des professeurs au 1er septembre 2017 à la Faculté de Médecine de Nice

\section{PRATICIEN HOSPITALIER UNIVERSITAIRE}

M. DURAND Matthieu

M. ILIE Marius

\section{PROFESSEURS ASSOCIÉS}

$\begin{array}{ll}\text { M. } & \text { GARDON Gilles } \\ \text { Mme } & \text { HURST Samia } \\ \text { M. } & \text { PAPA Michel }\end{array}$

\section{MAITRES DE CONFÉRENCES ASSOCIÉS}

M BALDIN Jean-Luc

Mme CASTA Céline

M. HOGU Nicolas

Mme MONNIER Brigitte
Urologie (52.04)

Anatomie et Cytologie pathologiques (42.03)
Médecine Générale (53.03)

Thérapeutique (48.04)

Médecine Générale (53.03)

Médecine Générale (53.03)

Médecine Générale (53.03)

Médecine Générale (53.03)

Médecine Générale (53.03)

\section{PROFESSEURS CONVENTIONNÉS DE L'UNIVERSITÉ}

$\begin{array}{ll}\text { M. } & \text { BERTRAND François } \\ \text { M. } & \text { BROCKER Patrice } \\ \text { M. } & \text { CHEVALLIER Daniel } \\ \text { Mme } & \text { FOURNIER-MEHOUAS Manuella } \\ \text { M. } & \text { JAMBOU Patrick } \\ \text { M. } & \text { ODIN Guillaume } \\ \text { M. } & \text { PEYRADE Frédéric } \\ \text { M. } & \text { PICCARD Bertrand } \\ \text { M. } & \text { QUARANTA Jean-François }\end{array}$

Médecine Interne

Médecine Interne Option Gériatrie Urologie

Médecine Physique et Réadaptation

Coordination prélèvements d'organes

Chirurgie maxilo-faciale

Onco-Hématologie

Psychiatrie

Santé Publique 


\section{TABLE DES MATIÈRES}

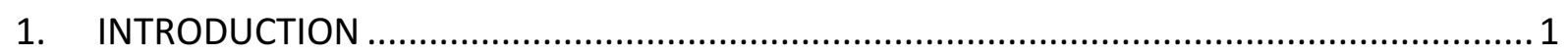

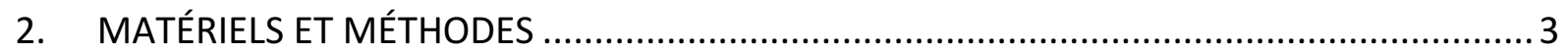

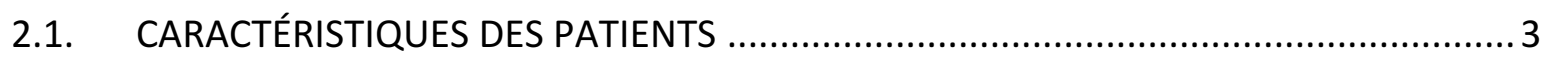

2.2. DONNÉES DE L'IRM MULTI-MODALE ............................................................ 4

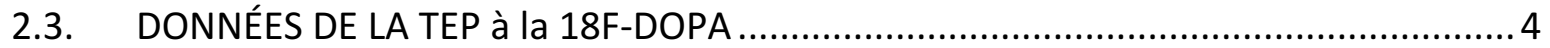

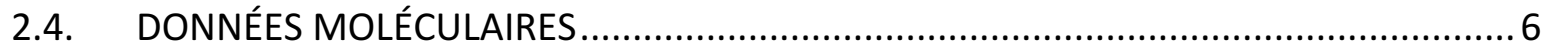

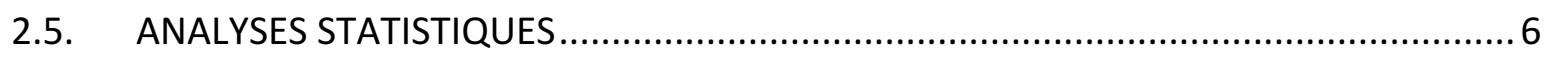

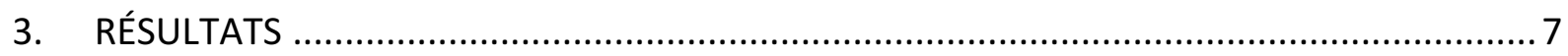

3.1. CARACTÉRISTIQUES TUMORALES ....................................................................... 7

3.2. COMPARAISON ENTRE LES RÉSULTATS DE LA TEP 18F-DOPA ET LES STATUTS

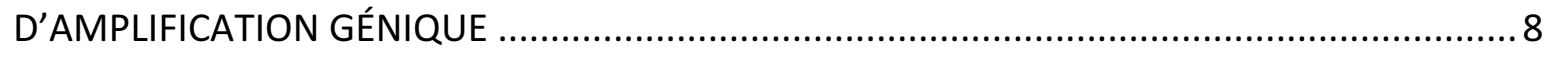

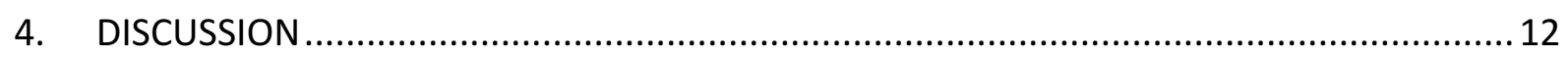

BIBLIOGRAPHIE

ANNEXES

ABRÉVIATIONS

SERMENT D'HIPPOCRATE

RESUMÉ 


\section{INTRODUCTION}

Les glioblastomes (GB) sont les tumeurs cérébrales primitives malignes les plus fréquentes chez l'adulte (1). Très agressifs, ils correspondent à des gliomes de grade IV selon la classification de l'Organisation Mondiale de la Santé (OMS) des tumeurs du système nerveux central. La dernière classification de l'OMS parue en 2016 distingue deux phénotypes moléculaires distincts de GB selon le statut des gènes isocitrate déshydrogénase (IDH) 1/2 (2) (annexe 1).

D'une part, les GB IDH non mutés sont majoritaires (>90\% des GB) et touchent des patients âgés (> 55 ans). Ils sont nommés " de novo " ou primaires car ils surviennent le plus souvent d'emblée sous la forme de grade IV. Ces GB sont ceux qui ont le plus mauvais pronostic.

D'autre part, les GB IDH mutés, plus rares ( $<10 \%$ des GB) affectent particulièrement le sujet jeune ( $<45$ ans). Ces sous-types sont dits secondaires car ils proviennent principalement de la transformation de gliomes de grades inférieurs (grades II et III).

Malgré les thérapeutiques actuelles basées principalement sur la chirurgie et la radiochimiothérapie (RCT), le pronostic de ces tumeurs reste très sévère. En effet, la médiane de survie est d'environ 15 mois $(3,4)$. En pratique, la récidive tumorale survient inéluctablement à quelques mois du traitement initial et peu de traitements sont efficaces en 2ème ligne.

L'imagerie par résonnance magnétique (IRM) est l'examen de référence pour le diagnostic et le suivi radiologique des tumeurs cérébrales. Au cours du suivi IRM, des phénomènes de pseudo-progression ou de pseudo-réponse peuvent rendre difficile le diagnostic différentiel entre récidive et remaniements induits par les traitements dont le plus fréquent est la radionécrose (5). En effet, les remaniements post-thérapeutiques peuvent augmenter la taille de la lésion prenant le produit de contraste, sans qu'il existe de véritable progression, on parle alors de pseudo-progression (6). A l'inverse, la mise sous traitement anti-angiogénique peut créer des aspects de pseudo-réponse (7). Au-delà des séquences d'IRM conventionnelles (T1 et T2), d'autres séquences d'IRM telles que la perfusion, la diffusion et/ou de spectroscopie sont alors pertinentes pour améliorer les performances diagnostiques (8-10).

Afin d'apporter des arguments complémentaires pour le diagnostic différentiel entre récidive tumorale et radionécrose, l'imagerie tomoscintigraphique par émission de positons (TEP) qui utilise des acides aminés ou des analogues d'acides aminés, avec parmi eux la 6-18F-fluoro-L3,4-dihydroxy-phenylalanine (18F-DOPA), est désormais recommandée en pratique clinique $(11,12)$. Dans la situation où l'IRM est d'interprétation litigieuse, la TEP à la 18F-DOPA permet de modifier la décision thérapeutique en réunion de concertation pluridisciplinaire (RCP) neuro-oncologique, dans près $30 \%$ des cas $(13,14)$. La suspicion de récidive tumorale est évaluée en pratique selon l'échelle proposée par Lizarraga (15). Le foyer captant la 18F-DOPA est considéré comme suspect de récidive lorsque son intensité est égale ou supérieure à celle du striatum controlatéral (annexe 2). Une analyse semi-quantitative permet de confirmer l'analyse visuelle lorsque l'intensité lésionnelle est proche de celle du striatum (16). Un seuil d'intensité maximal tumeur/striatum $\geq 1$ est rapporté comme ayant d'excellentes performances diagnostiques (17). 
Cependant, les mécanismes d'accumulation tumorale de ce radio-pharmaceutique restent mal connus. Selon des études in vitro, ils dépendraient principalement du transporteur d'acides aminés L-type amino-acid transporter 1 (LAT1)(18). D'autres facteurs biochimiques influenceraient ces mécanismes d'accumulation notamment la cible fonctionnelle de la rapamycine (mTOR)(19). Des phénomènes inflammatoires liés à la radiothérapie (RT) augmenteraient quant à eux l'intensité de captation des acides aminés évaluée en TEP (20). A l'échelle cellulaire, il a été montré que l'intensité de captation de la 18F-DOPA était corrélée au degré de prolifération tumorale évaluée par la mesure de l'index de prolifération (index Ki$67)$ ou au grade histologique (21-23).

Des publications récentes ont mis en évidence des associations entre des données d'imagerie métabolique et certains marqueurs génétiques comme la mutation IDH ou épigénétiques tel que la méthylation du promoteur de 06-methylguanine-DNA methyl-transferase (MGMT). Deux études publiées en 2016 et 2017, ont montré qu'une captation en 18F-FDOPA était plus élevée en cas de mutation IDH et de co-délétion 1p19q dans les sous-types de gliome de grade II et III (24,25). Dans une autre étude de 2017, les tumeurs qui présentaient une hyperméthylation du promoteur de MGMT avaient des volumes tumoraux initiaux inférieurs à celles qui ne possédaient pas cette anomalie épigénétique (26).

Ainsi, les données de la littérature suggèrent que les données des TEP aux acides-aminés pourraient varier en fonction des anomalies moléculaires présentes dans les GB.

Les amplifications géniques sont fréquentes dans le GB et participent à leur grande hétérogénéité. Elles interviennent dans de multiples voies de signalisation tumorales cruciales pour la survie cellulaire, la prolifération, l'invasion et l'angiogenèse (27) (annexe 3). L'amplification du gène codant pour le récepteur epidermal growth factor (EGFR) est la plus commune. Elle est retrouvée dans près de $40 \%$ des cas de GB IDH sauvage (28). D'autres sont plus rares et impliquent des gènes codant pour les récepteurs mesenchymal-epithelial transition (MET), alpha platelet-derived growth factor (PDGFRA) ou pour cyclin-dependent kinase 4 (CDK4), murine double minute 2 (MDM2) ou murine double minute 4 (MDM4).

En dérégulant les voies de signalisation qui conduisent à la prolifération cellulaire, où la captation d'acides aminés est accrue, les amplifications géniques pourraient avoir un impact sur les mécanismes d'accumulation des acides-aminés utilisés en TEP.

A notre connaissance, une seule étude, réalisée en pré-thérapeutique, s'est intéressée au lien entre l'amplification EGFR et l'imagerie TEP en utilisant un radio-pharmaceutique acide aminé non utilisé en routine, l'alpha[C-11] -L-methyl-tryptophane (AMT). Dans cette étude, les volumes tumoraux en TEP/IRM apparaissaient inversement corrélés au statut d'amplification de EGFR.

L'objectif de notre travail a été de rechercher chez les patients atteints d'un GB primaire en situation de récidive si les résultats de la TEP à la 18F-DOPA étaient influencés par le statut d'amplification génique de ces tumeurs. 


\section{MATÉRIELS ET MÉTHODES}

\subsection{CARACTÉRISTIQUES DES PATIENTS}

L'ensemble des patients participait à l'essai clinique " IMOTEP » (RECF2013) ou faisait partie du programme hospitalier de recherche clinique " POSEIDON » ( $n^{\circ} 14-061$; FINESS juridique 060000528).

Quatre-vingt-treize patients avec une tumeur cérébrale ont réalisé une TEP à la 18F-DOPA au Centre Antoine Lacassagne à Nice entre 2013 et 2017. Les critères d'éligibilité pour l'analyse étaient : patients majeurs, atteints d'un GB hémisphérique histologiquement prouvé, IDH non muté et dont le statut d'amplification génique était connu (figure 1).

Au total, 31 patients adultes (54,8\% homme et $45,2 \%$ femme ; âge moyen 59,3 ans) ayant un GB suspects de récidive à la fois en IRM multi-modale et en TEP à la 18F-DOPA ont été rétrospectivement inclus dans cette étude entre janvier 2013 et janvier 2017.

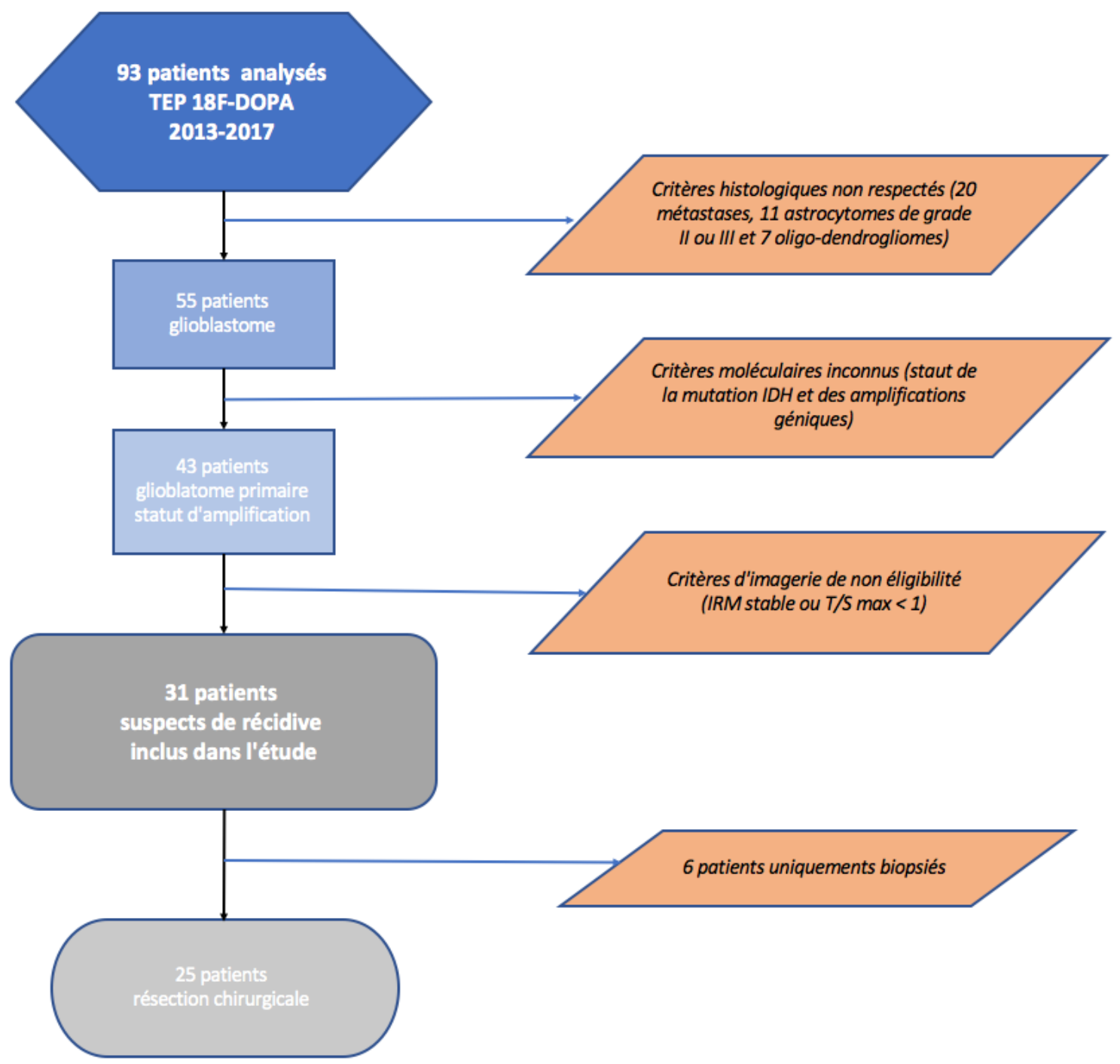

Figure 1. Diagramme de flux. 
Deux types de délai de récidive ont été définis, l'un entre la date de la chirurgie et la date de l'examen TEP et l'autre entre la fin de la RT et la date de l'examen TEP.

\subsection{IRM MULTI-MODALE}

\section{PROTOCOLE D'ACQUISITION}

Les IRM étaient réalisées sur des appareils General Electric healthcare 1,5T, à l'aide d'une antenne cérébrale (haute densité d'éléments).

Le protocole de l'examen comprenait les séquences conventionnelles (axiales T2, sagittales 3D FLAIR, axiales 3D T1 sans et après injection de chélates de gadolinium), fonctionnelles (séquences de perfusion DSC T2* ; séquences de diffusion gradient $b=0$ et $b=1000$ ) et métaboliques (séquences de spectroscopie mono-voxel à TE court).

La durée totale d'acquisition était d'environ 40 minutes.

Le post-traitement pour la visualisation des images était réalisé sur des consoles GE avec le logiciel Advantage Workstation (version 4.6).

\section{ANALYSE DES IMAGES}

Les critères de suspicion de récidive tumorale en IRM étaient basés sur la classification RANO (annexe 4), sur les séquences de perfusion (volume sanguin cérébral relatif $>2$ ), de spectroscopie (inversion du pic N-Acétyl-Aspartate/Choline) et sur la cartographie du coefficient apparent de diffusion (ADC).

L'intervalle de temps entre l'examen TEP et l'examen IRM était en moyenne de 11,5 jours avec un maximum de 42 jours. L'IRM était comparée à l'IRM de référence post-opératoire précoce. Les IRM de suivi, jusqu'à 3 mois après l'examen TEP/IRM, permettaient également de préciser le statut de récidive. Les patients dont l'IRM était stable ou en réponse partielle étaient exclus de l'étude.

\subsection{TEP à la 18F-DOPA}

\section{PROTOCOLE D'ACQUISITION}

La TEP à la 18F-DOPA était effectuée au cours du suivi post-thérapeutique sur un appareil TEP/TDM Biograph mCT (Siemens Healthineers, Erkangen, Germany). Chaque patient recevait une prémédication par Carbidopa 100 mg 1 heure avant l'injection du radio-pharmaceutique, en l'absence de contre-indication.

Les acquisitions de 10 minutes étaient réalisées 20 minutes après injection de $2 \mathrm{MBq} / \mathrm{kg}$ de 18F-DOPA. Les images d'émission étaient reconstruites en utilisant l'algorithme OSEM (5 itérations et 24 sous-ensembles), avec correction de l'atténuation, du diffusé, des fortuits mais sans correction de la réponse impulsionnelle ni du temps de vol.

La visualisation des images était faite sur une console Siemens avec le serveur SyngoVia.

\section{ANALYSE DES IMAGES}

Le critère définissant la récidive était un seuil d'intensité maximale tumeur/striatum ( $T / \mathrm{S}$ max) supérieur ou égal à 1.

L'intensité de captation de la 18F-DOPA était déterminée en utilisant une méthode semiquantitative. Les valeurs de Standard Uptake Value (SUV) étaient corrigées par le poids du 
patient $(b w)$. Les valeurs de SUV maximale dans un pixel (SUV max) et de SUV moyen dans une sphère de $1 \mathrm{~cm}^{3}$ centrée sur le maximum (SUV " peak ») étaient obtenues au niveau de la lésion $(T)$, du striatum controlatéral $(S)$ et de l'hémisphère sain controlatéral $(N)$. Ainsi, nous avons déterminé 4 valeurs d'intensités relatives pour chaque patient : T/S max, T/S peak, T/N max, $\mathrm{T} / \mathrm{N}$ peak.

Le SUV moyen de I'hémisphère sain controlatéral (SUV mean N) était obtenu à l'aide d'une sphère automatisée de $7 \mathrm{~mm}$ de rayon $\left(\sim 14 \mathrm{~cm}^{3}\right)$ située à la frontière substance blanche/substance grise, en évitant les ventricules et le tissu sous-cutané.

Deux valeurs de SUV moyen du striatum (SUV mean S) ont été calculées par iso-contour : à $80 \%$ et à $60 \%$ du maximum.

Les volumes biologiques ont alors été calculés en prenant des seuils par rapport au striatum et par rapport à l'hémisphère sain. Un volume $1.6 \mathrm{~N}$ (Vol $1.6 \mathrm{~N})$, le plus grand des volumes calculés, contenait toutes les valeurs à partir de 1.6 fois la moyenne de l'hémisphère sain. Deux autres volumes, calculés par rapport au striatum (Vol $0.8 \mathrm{~S}$ et Vol0.6S), avaient pour seuils respectifs les valeurs moyennes de 80 et $60 \%$ du SUV max.

$\mathrm{Au}$ total, 3 volumes biologiques tumoraux ont été calculés : Vol $1.6 \mathrm{~N}$, Vol $0.8 \mathrm{~S}$, Vol $0.6 \mathrm{~S}$ (figure 2).
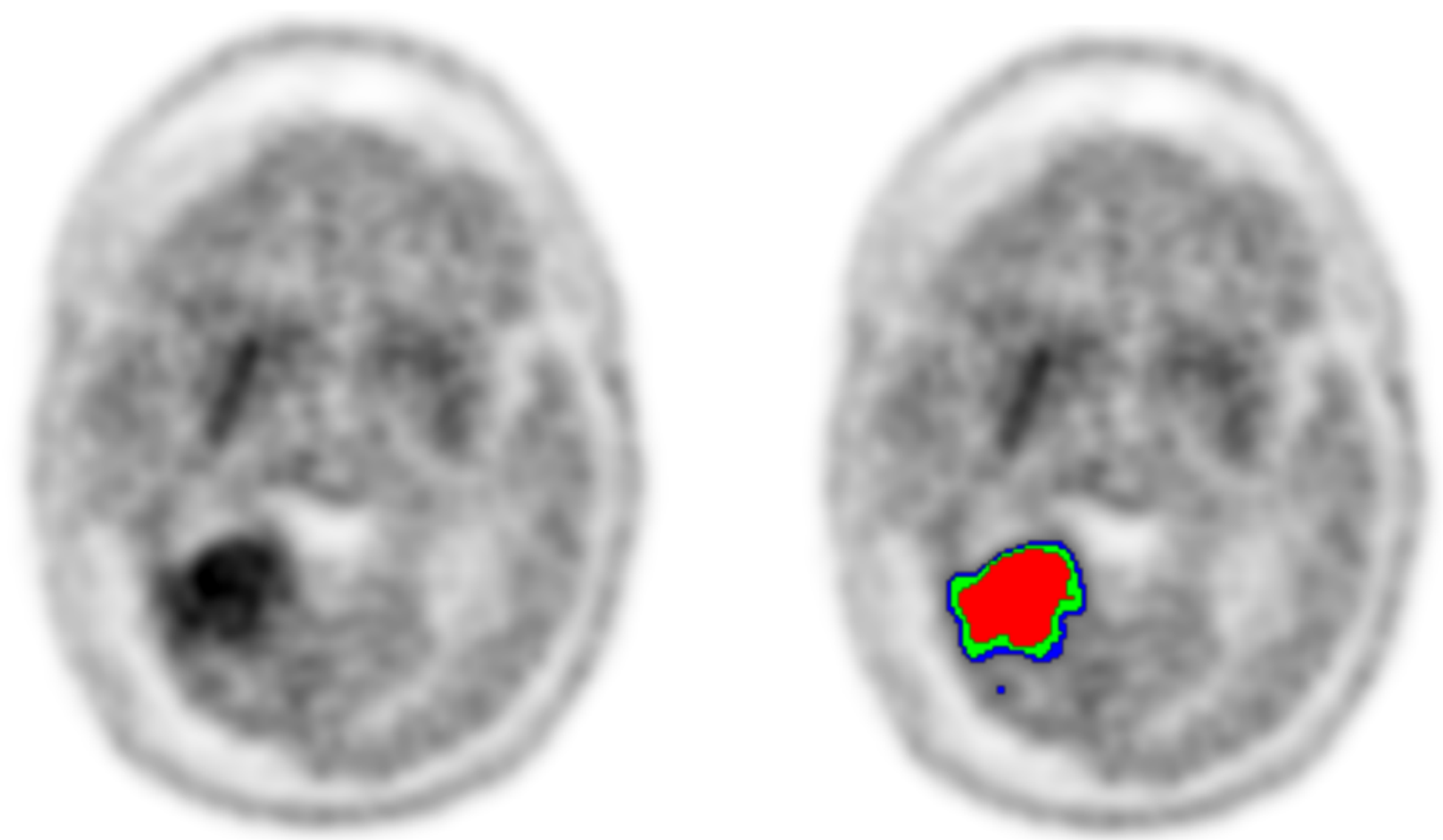

Figure 2. Représentation de la segmentation tumorale selon 3 seuils différents (la moyenne de 0.8 du SUV max du striatum, la moyenne de 0.6 du SUV max du striatum et le SUV moyen de l'hémisphère sain multiplié par un facteur 1.6). Patient de 41 ans avec une récidive de GB située à la partie postéro-supérieure de la cavité opératoire temporale droite. Les seuils respectifs $\left(3,4 ; 2,9\right.$; et 2,6) nous ont permis de segmenter la lésion en 3 volumes biologiques tumoraux différents (rouge : $6 \mathrm{~cm}^{3}$, vert : $14 \mathrm{~cm}^{3}$ et bleu : $20 \mathrm{~cm}^{3}$ ). 


\subsection{DONNÉES MOLÉCULAIRES}

Tous les patients étaient atteints d'un GB histologiquement prouvé, IDH non muté. La mutation était recherchée par immuno-histochimie (IHC) pour tous les patients et par Next Generation Sequencing (NGS) oncopanel pour ceux de moins de 55 ans, selon les recommandations actuelles.

L'étude immunohistochimique était réalisée sur coupe de $2 \mu \mathrm{m}$, avec l'anticorps dirigé contre la forme mutée IDH1 R132H (clone H09, Abnova, 1/100), en utilisant l'automate de Dako (Glostrup, Denmark), selon les recommandations du fabricant. L'étape de démasquage était effectuée avec le kit « Envision Flex » de Dako.

Pour le NGS, l'ADN génomique était extrait à partir du tissu fixé au formol et inclus en paraffine (FFPE), en utilisant un système Maxwell (Promega). Le séquençage était réalisé en utilisant la technologie semi-conducteur Ion Torrent (Life Technologies, Grand Island, NY) pour cibler 50 oncogènes et gènes suppresseurs de tumeurs incluant IDH1 et IDH2 (Ion AmpliSeq Cancer Hotspot Panel v2, Life Technologies).

Le statut d'amplification génique était recherché par la technique d'hybridation génomique comparative sur puce à ADN Comparative Génomic Hybridization (CGH array). L'ADN tumoral était extrait à partir de tissu fixé au formol et inclus en paraffine, avec le système Qiamp DNA mini kit (Qiagen, Courtaboeuf, France) après déparaffinage dans une solution de Tween 20, sans xylène. L'ADN témoin non tumoral de référence était fourni par Agilent Technologies (Santa Clara, CA). L'ADN génomique du patient était marqué avec une molécule fluorescente, la Cyanine 5 (Cy5) et l'ADN témoin avec la Cyanine 3 (Cy3) (Genomic DNA SureTag Labeling Kit, Agilent). Les ADN étaient co-hybridés sur une puce à ADN (Sureprint G3 Human CGH microarray 4×180K, Agilent). La lame était scannée (SureScan, Agilent) et les images analysées par un logiciel (Cytogenomics v2.5.8.11, Agilent) capable de convertir des ratios de fluorescence sous forme de tracés de Log (2) : Cy5/Cy3. Les résultats étaient exprimés selon la référence hg19 (GRCh37 Genome Reference Consortium Human Reference 37). Les amplifications géniques étaient définies par un log ratio cya5/cya3 $>1.8$

\subsection{ANALYSES STATISTIQUES}

Des statistiques descriptives ont été réalisées pour définir les caractéristiques démographiques cliniques (âge, sexe, traitement anti-angiogénique), chirurgicales (biopsie, résection totale, résection partielle), moléculaires (amplifications géniques dont EGFR) et les données temporelles (délais de récidive).

Les variables quantitatives ont été décrites par la moyenne, l'écart type et les quartiles. Elles ont été comparées à l'aide de test $T$ de Student ou de test de $U$ de Mann Whitney en cas de non-respect des conditions d'application.

Les variables qualitatives ont été décrites en fréquence absolue et relative. Elles ont été comparées à l'aide de test de Chi2 ou de test de Fisher en cas de non-respect des conditions d'application.

Les statuts d'amplification génique (amplifiés ou non amplifiés) ont été comparés aux résultats de la TEP (intensités et volumes) en fonction de la présence d'au moins une amplification ou de la présence plus spécifiquement de l'amplification de EGFR. Ces 
comparaisons étaient effectuées en utilisant le t-test de Student ou le test U de Mann Whitney si les conditions du test paramétrique n'étaient pas remplies.

Une analyse multivariée ajustée sur l'âge a permis de comparer l'intensité (T/S max) avec le statut d'amplification génique.

Une analyse en sous-groupe, sur 25 patients traités par résection, a été réalisée afin d'avoir une meilleure comparabilité des volumes tumoraux. Les comparaisons entre le statut d'amplification de $E G F R$, les volumes, l'âge, le type de résection et les délais de récidive ont été réalisées à l'aide du t-test ou le test $U$ si les conditions du test paramétrique n'étaient pas remplies.

Les analyses statistiques ont été réalisées en utilisant le logiciel SPSS (20.0; IBM).

Tous les tests statistiques ont été réalisés en hypothèse bilatérale avec un seuil de significativité de $5 \%$.

\section{RÉSULTATS}

\subsection{CARACTÉRISTIQUES DEMOGRAPHIQUES}

Sur une population de 55 patients atteints de GB, suivis par IRM et TEP à la 18F-DOPA après traitement initial, 31 cas de GB primaires (IDH non mutés) ont été retenus comme récidive sur les données combinées de l'IRM multimodale et de la TEP à la F-DOPA.

Vingt-cinq patients avaient bénéficié d'une résection tumorale initiale. La résection était considérée comme totale sur l'IRM chez 8 d'entre eux (absence de reliquat significatif sur la séquence $\mathrm{T} 1$ injectée à 24-72 heures post-opératoire). Les délais de récidive étaient retardés lorsque la résection chirurgicale était totale (en moyenne, 294 jours contre 197 jours pour ceux présentant une résection partielle).

Les 6 autres patients avaient eu une biopsie diagnostique.

$25 \%$ des patients (8/31) étaient sous traitement anti-angiogénique lors de l'examen TEP/IRM. $35 \%(11 / 31)$ des patients ont été traités par reprise chirurgicale après la TEP, avec chez tous ces patients la confirmation histologique du diagnostic radiologique de récidive.

L'amplification de EGFR, était détectée dans 10/31 des tumeurs (32\%),

Les autres amplifications géniques (PDGRA, CDK4, MDM2, MDM4, MET) étaient chacune présentes dans moins de $20 \%$ des cas. 6 GB sur 31 présentaient des coamplifications (EGFR+PDGFRA+CDK4+MDM2 ; EGFR+MDM4; PDGFRA+CDK4+MDM2 ; PDGFRA+MDM2; PDGFRA+CDK4+MET;CDK4+MDM2).

Les fréquences des amplifications géniques sont précisées dans le tableau 1. 
Tableau 1. Fréquences des amplifications géniques.

\begin{tabular}{ll}
\hline Amplifications & $\mathbf{1 8 / 3 1}(\mathbf{5 8 , 1} \%)$ \\
\hline EGFR & $\mathbf{1 0 / 3 1 ( 3 2 , 3} \%)$ \\
\hline PDGFRA & $4 / 31(12,9 \%)$ \\
\hline CDK4 & $5 / 31(16,1 \%)$ \\
\hline$M D M 2$ & $4 / 31(12,9 \%)$ \\
\hline$M D M 4$ & $2 / 31(6,5 \%)$ \\
\hline$M E T$ & $3 / 31(9,7 \%)$ \\
\hline
\end{tabular}

\subsection{COMPARAISON ENTRE LES RÉSULTATS DE LA TEP 18F-DOPA ET LES STATUTS D'AMPLIFICATION GÉNIQUE}

Nous avons cherché si les données de la TEP à la 18F-DOPA étaient corrélées au statut d'amplification génique.

\section{INTENSITES}

Nous avons mis en évidence une corrélation inverse entre les valeurs d'intensités relatives maximales ( $T / \mathrm{S}$ et $\mathrm{T} / \mathrm{N}$ ) et les statuts d'amplification génique.

Les intensités relatives étaient plus importantes chez les patients ne présentant aucune amplification (tableau 2) (en moyenne T/S max : 1,3 +/- 0,3 vs $1,2+/-0,2 ; p=0,031$ ).

II n'y avait pas de différence significative d'intensité entre les GB EGFR amplifiés et les GB non amplifiés pour EGFR ( $p=0.14$ pour l'intensité T/S max).

Les patients plus âgés présentaient plus d'amplification génique (en moyenne 63,2 +/- 7,9 ans dans le groupe amplification et $53,8+/-11,2$ ans dans le groupe ne présentant aucune amplification ; $p=0,01$ ).

En analyse multivariée, la corrélation inverse entre le statut d'amplification génique et les résultats des intensités de captation était dépendant de l'âge des patients $(p=0.066)$.

VOLUMES

Nous avons mis en évidence une corrélation inverse entre les volumes calculés par rapport au striatum (Vol 0.8S et Vol 0.6S) et le statut d'amplification de EGFR. Les GB qui présentaient une amplification de EGFR avaient des volumes biologiques tumoraux plus petits que les $G B$ non amplifiés EGFR (médiane arrondie à l'unité, Vol $0.6 \mathrm{~S}: 3 \mathrm{~cm}^{3}$ [2- 8] vs $14 \mathrm{~cm}^{3}$ [9-27]; $p=$ 0.029) (tableau 3).

Le type de chirurgie pratiquée (résection vs biopsie) pouvant faire varier les volumes, nous avons vérifié ces résultats sur le sous-groupe des GB traités par résection. La corrélation inverse entre les volumes et le statut d'amplification EGFR s'est confirmée $(p=0.022)$ (tableau 4)

Les volumes moyens définis à partir de l'hémisphère sain ( $\mathrm{Vol} 1.6 \mathrm{~N})$ ne présentaient pas de différence significative entre les GB EGFR amplifiés et les GB EGFR non amplifiés ( $p=0.183$ ). 
Un exemple des résultats volumiques est représenté sur la figure 3 en fonction du statut EGFR amplifié ou non.

Les volumes tumoraux avaient tendance à être plus petits chez les patients qui présentaient au moins une amplification ( $p=0.056$ pour le volume $0.8 \mathrm{~S}$ ). Les résultats étaient similaires dans le sous-groupe des GB traités initialement par résection chirurgicale.

Les délais de récidive $n$ 'apparaissaient pas corrélés aux volumes tumoraux (en moyenne, pour le vol $0.8 \mathrm{~S}>3 \mathrm{~cm} 3$ récidive à 220 jours et pour celui $<$ à $3 \mathrm{~cm} 3$ récidive à 277 jours lorsque l'on prend comme point de départ la date de la chirurgie) ni au statut EGFR (délai moyen de récidive pour les GB EGFR amplifiés de 292 jours contre 233 jours pour les GB EGFR non amplifiés ; $p=0,72$ ).

Au total, les amplifications géniques étaient associées à des modifications de captation du radio-pharmaceutique lors de la récidive tumorale, comparativement aux GB sans amplification : les GB présentant au moins une amplification génique étaient associés à des intensités de captation plus faibles et les GB EGFR amplifiés étaient corrélées à des volumes biologiques tumoraux plus petit.

Tableau 2. Comparaison des données de la TEP à la 18F-DOPA en fonction de la présence d'au moins une amplification génique.

\begin{tabular}{llcc} 
Patients $(n=31)$ & \multicolumn{3}{c}{ AMPLIFICATION } \\
\cline { 2 - 3 } & Oui $(n=18)$ & Non $(n=13)$ & 0,031 \\
\hline $\begin{array}{c}\text { T/S max } \\
\text { Moyenne (écart-type) }\end{array}$ & $1,2(0,2)$ & $1,3(0,3)$ & 0,038 \\
\hline $\begin{array}{l}\text { T/S peak } \\
\text { Moyenne (écart-type) }\end{array}$ & $1,2(0,2)$ & $1,4(0,3)$ & 0,037 \\
\hline $\begin{array}{l}\text { T/N max } \\
\text { Moyenne (écart-type) }\end{array}$ & $2,1(0,2)$ & $2,3(0,4)$ & 0,1 \\
\hline $\begin{array}{l}\text { T/N peak } \\
\text { Médiane (étendue) }\end{array}$ & $2,0(1,8 ; 2,3)$ & $2,3(2,1 ; 2,4)$ & \\
\hline & & 0,056 \\
\hline $\begin{array}{l}\text { Vol 0.8S, en cm } \\
\text { Médiane (étendue) }\end{array}$ & $2,1(0,2 ; 4,1)$ & $7,9(3,9 ; 15)$ & 0,101 \\
\hline $\begin{array}{l}\text { Vol 0.6S, en } \mathrm{cm}^{3} \\
\text { Médiane (étendue) }\end{array}$ & $6,9(2 ; 13,6)$ & $14,4(8,7 ; 27)$ & 0,207 \\
\hline $\begin{array}{l}\text { Vol 1.6N, en } \mathrm{cm}^{3} \\
\text { Médiane (étendue) }\end{array}$ & $15,5(6,8 ; 31,2)$ & $27,5(18,6 ; 38,4)$ \\
\hline
\end{tabular}


Tableau 3. Comparaison des données de la TEP à la 18F-DOPA en fonction de la présence d'une amplification de EGFR

\begin{tabular}{|c|c|c|c|}
\hline \multirow[t]{2}{*}{ Patients ( $n=31$ ) } & \multicolumn{3}{|c|}{ AMPLIFICATION EGFR } \\
\hline & Oui $(n=10)$ & Non $(n=21)$ & $p$ \\
\hline \multicolumn{4}{|l|}{$\mathrm{T} / \mathrm{S} \max$} \\
\hline Moyenne (écart-type) & $1,1(0,2)$ & $1,3(0,2)$ & 0,14 \\
\hline \multicolumn{4}{|l|}{$\mathrm{T} / \mathrm{S}$ peak } \\
\hline Moyenne (écart-type) & $1,1(0,2)$ & $1,3(0,3)$ & 0,145 \\
\hline \multicolumn{4}{|l|}{$\mathrm{T} / \mathrm{N} \max$} \\
\hline Médiane (étendue) & $2(1,9 ; 2,2)$ & $2,2(1,9 ; 2,3)$ & 0,386 \\
\hline \multicolumn{4}{|l|}{$\mathrm{T} / \mathrm{N}$ peak } \\
\hline Médiane (étendue) & $2(1,8 ; 2,3)$ & $2,2(1,9 ; 2,3)$ & 0,3 \\
\hline \multicolumn{4}{|l|}{ Vol $0.8 \mathrm{~S}$, en $\mathrm{cm}^{3}$} \\
\hline Médiane (étendue) & $0,9(0,2 ; 3)$ & $6,4(1,8 ; 12,5)$ & 0,042 \\
\hline \multicolumn{4}{|l|}{ Vol $0.6 \mathrm{~S}$, en $\mathrm{cm}^{3}$} \\
\hline Médiane (étendue) & $3,3(1,7 ; 8,3)$ & $14,1(8,7 ; 27)$ & 0,029 \\
\hline \multicolumn{4}{|l|}{ Vol $1.6 \mathrm{~N}$, en $\mathrm{cm}^{3}$} \\
\hline Médiane (étendue) & $9,9(5,1 ; 31,2)$ & $20(14,9 ; 38,4)$ & 0,183 \\
\hline
\end{tabular}

Tableau 4. Comparaison des volumes biologiques tumoraux en fonction du statut EGFR dans le sous-groupe des résections.

\begin{tabular}{lccc} 
Patients $(n=25)$ & \multicolumn{3}{c}{ AMPLIFICATION EGFR } \\
\cline { 2 - 4 } & Oui $(\mathrm{n}=9)$ & Non $(\mathrm{n}=16)$ & $p$ \\
\hline $\begin{array}{l}\text { Vol 0.8S, en } \mathrm{cm}^{3} \\
\text { Médiane (étendue) }\end{array}$ & $0,6(0,2 ; 2,5)$ & $4,3(1,5 ; 9,3)$ & $\mathbf{0 , 0 3 5}$ \\
\hline $\begin{array}{l}\text { Vol 0.6S, en cm } \\
\text { Médiane }\end{array}$ & $2,6(1,6 ; 4,2)$ & $12,6(7,3 ; 17,1)$ & $\mathbf{0 , 0 2 2}$ \\
\hline $\begin{array}{l}\text { Vol 1.6N, en cm } \\
\text { Médiane }\end{array}$ & $6,4(4,7 ; 29)$ & $19,4(14,4 ; 29,2)$ & 0,165 \\
\hline
\end{tabular}




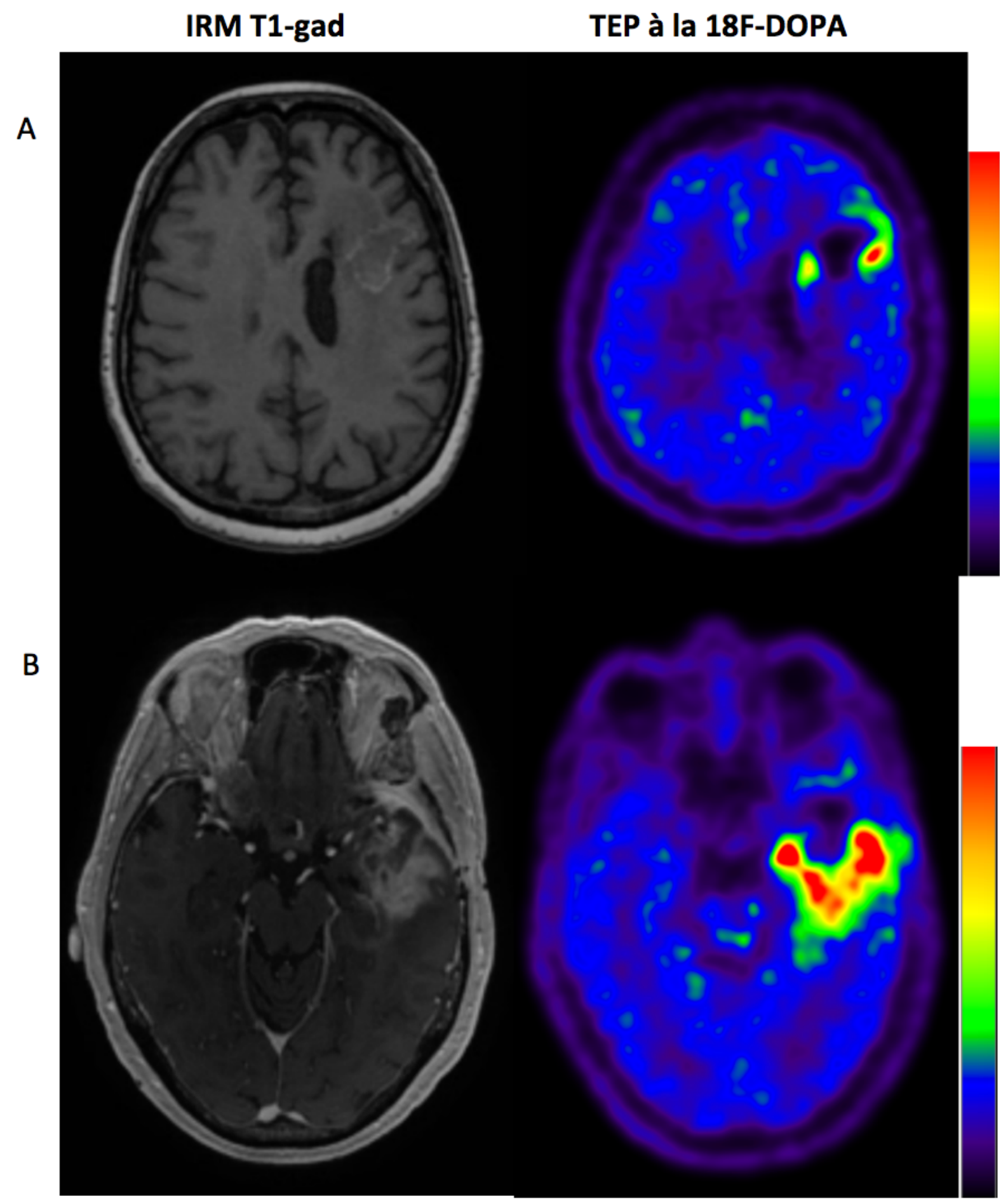

Figure 3. Exemple de volumes en la TEP à la 18F-DOPA qui sont inversement corrélés au statut d'amplification de EGFR. A : GB frontal gauche EGFR amplifié. Vol $0.6 \mathrm{~s}=2,6 \mathrm{~cm}^{3} ; \mathbf{B}: \mathrm{GB}$ temporal gauche $E G F R$ non amplifié. $\mathrm{Vol}_{0.6 \mathrm{~S}}=18.4 \mathrm{~cm}^{3}$. 


\section{DISCUSSION}

Bien que la TEP à la 18F-DOPA occupe une place croissante dans le suivi des glioblastomes (GB), ses mécanismes d'accumulation dans les tumeurs cérébrales sont encore mal connus. Les amplifications géniques, anomalies fréquentes dans les GB primaires, dérégulent les voies de signalisation intracellulaires et confèrent aux cellules des propriétés de prolifération, $d$ 'invasion et d'angiogenèse.

Nos résultats montraient que les amplifications géniques influençaient la captation de la 18FDOPA lors de la récidive tumorale, comparativement aux GB sans amplification : les GB présentant au moins une amplification génique étaient associés à des intensités de captation plus faibles et les GB EGFR amplifiés étaient corrélés à des volumes biologiques tumoraux plus petits.

Notre travail a été réalisé sur une population homogène et ciblée. Nous avons sélectionné exclusivement la forme de GB la plus fréquente, les GB primaires, définis par l'absence de mutation des gènes $I D H 1 / 2$. La récidive tumorale pouvant être difficile à distinguer des remaniements post-thérapeutiques en pratique, nous avons inclus les patients pour lesquels la récidive tumorale était certaine. Nous avons retenu des critères de sélection stricts en IRM multi-modale et en TEP à la 18F-DOPA. Pour la TEP, le seuil pour définir la récidive était T/S $\max \geq 1$. Il présente la meilleure spécificité de détection des lésions cérébrales d'après l'étude de Chen et al. (17). De plus, les données de la littérature utilisent fréquemment ce seuil pour différencier récidive et radionécrose (29-31). Parmi les 93 patients qui avaient bénéficié d'une TEP à la 18F-DOPA cérébrale entre 2013 et 2017, nos critères d'éligibilité des patients dans l'étude ont eu comme corollaire de réduire la taille de l'échantillon analysé à 31 cas. Le traitement initial différent (résection ou biopsie) pouvait influencer les volumes tumoraux en récidive, ce qui nous a conduit à mener une analyse en sous-groupes. Bien que réduisant la puissance statistique de l'étude, l'analyse en sous-groupes nous a permis de vérifier que les volumes tumoraux lors de la récidive étaient indépendants des facteurs de confusion comme le type de résection chirurgicale et le délai de récidive. Le caractère rétrospectif et l'absence de TEP de référence pré-thérapeutique étaient également des limites à notre étude.

De plus, parmi les 3 volumes biologiques calculés, le volume défini par rapport au SUV moyen de l'hémisphère sain ( $\mathrm{Vol} 1.6 \mathrm{~N}$ ) ne montrait pas de différence significative en fonction de I'amplification de EGFR. Un biais de mesure pourrait expliquer ce résultat car nous avons constaté des différences de valeurs moyennes selon la localisation du volume d'intérêt (VOI) sur l'hémisphère. Cela suggère que le volume Vol $1.6 \mathrm{~N}$ serait dépendant de la localisation du VOI choisi. Rétrospectivement, l'impact de ce biais potentiel aurait pu être réduit en calculant la moyenne de plusieurs VOI rapprochées sur l'hémisphère sain (32), en tenant compte des déviations standards (33), ou bien en définissant une localisation hémisphérique fixe pour les mesures de SUV moyens. Enfin, nous pouvions noter un biais potentiel lié à l'éventuelle modification dans le temps du statut des amplifications géniques analysé en post-opératoire initial et non connu lors de la récidive. Ce biais semblerait restreint d'après une publication récente qui montrait que le statut d'amplification génique était relativement stable dans le temps (34).

Notre travail est novateur car il s'agit, à notre connaissance, de la première étude en TEP à la 18F-DOPA cherchant une corrélation entre l'accumulation de ce radio-pharmaceutique et les amplifications géniques dans les GB primaires en situation de récidive. 
Une seule étude antérieure, de Bosnyak et al. (26) s'intéressait au lien entre les amplifications géniques et les résultats de la TEP aux acides aminés dans les glioblastomes primaires. Dans cette étude, les données volumiques étaient recueillies en pré-thérapeutique et le radiopharmaceutique utilisé était l'alpha[C-11] -L-methyl-tryptophane (AMT). Ces résultats, ne trouvaient pas de différence significative de volume en TEP-AMT selon le statut de EGFR mais, à l'inverse de la 18F-DOPA, I'AMT est métabolisé par des voies enzymatiques (kynurenine). De plus, cet article objectivait que les GB EGFR amplifiés étaient associés à des volumes en IRM sur la séquence T1-gadolinium (gad) et à des ratios de volumes T1-gad/TEP plus faibles que pour les GB sans amplification. Les auteurs émettaient l'hypothèse que les GB amplifiés EGFR seraient plus infiltrants et plus angiogéniques. Ces arguments ne sont pas suffisants pour expliquer scientifiquement leurs résultats.

Nos résultats mettaient en évidence que les $G B$ récidivants avec amplification de $E G F R$ avaient des volumes biologiques tumoraux inférieurs comparés à ceux des $G B$ sans amplification. Nous avons d'abord été surpris par ces résultats car la surexpression de EGFR, induite par l'amplification du gène est connue pour promouvoir l'invasion, la prolifération et l'angiogénèse. Ces résultats impliquaient à l'inverse que les GB sans amplification EGFR avaient des volumes tumoraux supérieurs comparés à ceux des GB avec amplification. Cela suggère que les GB sans amplification de EGFR seraient métaboliquement plus agressifs que les GB EGFR amplifiés lors de la récidive. De plus, les GB primaires (IDH non mutés) sont un groupe moléculaire hétérogène. Parmi les différents sous-types moléculaires de GB identifiés par Verhaak et al. (35) (annexe 5), certains sont dépourvus de l'amplification de EGFR, comme les GB « mésenchymateux » décrits comme particulièrement agressifs $(36,37)$. Nos résultats sembleraient donc s'inscrire dans les connaissances actuelles de la biologie tumorale.

Notre étude montrait également que la présence " d'au moins une amplification génique » était corrélée à de plus faibles intensités de captation de la 18F-DOPA par rapport à celles des GB sans amplification génique. Ces résultats suggèrent que l'absence d'amplification génique, dans les GB primaires récidivants pourrait aussi être un facteur de plus grande agressivité métabolique. Les GB sans amplification auraient des besoins accrus en acide aminé. En revanche, en prenant uniquement le statut d'amplification de EGFR nous ne mettons pas en évidence de corrélation avec les ratios des intensités. Ceci pourrait être lié à un manque de puissance statistique dans notre étude (10/31 GB étaient EGFR amplifiés alors que 18/31 avaient au moins une amplification).

La recherche de corrélation entre les anomalies moléculaires de EGFR et les données de TEP ont été effectuées dans d'autres types de cancer, notamment le cancer pulmonaire non à petits cellules (CPNPC). Les résultats montraient que la présence d'une mutation de EGFR était associée à une réduction du métabolisme cellulaire, en particulier glucidique. Dans l'étude de Yip et al.(38) la présence d'une mutation du gène EGFR, était associée, en pré-thérapeutique, à de petits volumes métaboliques tumoraux en TEP 18F-fluoro-2-deoxy-glucose (18FDG) en comparaison aux volumes des tumeurs EGFR non mutés. Une autre publication récente trouvait des résultats similaires (39). De la même manière, Takamoshi et al. (40) ont objectivé que parmi les adénocarcinomes pulmonaires, les intensités de captation en TEP-18FDG des tumeurs EGFR mutés étaient inférieures à celle des tumeurs EGFR non mutées. Cette différence était liée à une réduction du métabolisme du glucose en cas de mutation de EGFR. 
Dans la littérature, concernant les CPNPC, nous n'avons pas trouvé de données sur le métabolisme des acides aminés en TEP selon le statut de EGFR. Notre étude, sur les GB, faisait apparaître une réduction de ce métabolisme en cas de dérégulation de $E G F R$. De nouveaux travaux corrélant les anomalies moléculaires et les données de la TEP ainsi que des études fondamentales serraient nécessaires pour mieux appréhender le lien entre les voies métaboliques et le statut du gène EGFR.

En ce qui concerne les facteurs pronostiques des GB, l'âge est le plus important (41). La survie des patients âgés de plus de 60 ans est inférieure à celle des plus jeunes. Dans notre étude, les patients âgés présentaient davantage d'amplifications géniques mais le statut d'amplification de EGFR était indépendant de l'âge. L'amplification du gène codant pour EGFR, la plus fréquente dans les GB primaires, est associée à une augmentation de la prolifération cellulaire. Cependant, la valeur pronostique de la dérégulation de EGFR reste controversée car les résultats des études de survie sont contradictoires. Les résultats d'une méta-analyse sur 8 articles de Chen et al (42) indiquaient un manque évident de preuve inhérent au potentiel pronostique des anomalies moléculaires de EGFR dans les GB. D'autres articles ont néanmoins montré des valeurs pronostiques favorables en présence d'anomalies de EGFR. Une étude prospective de Montano et al.(43) mettait en évidence que la mutation du variant III de EGFR, forme tronquée constitutionnellement active présente dans environ $20 \%$ des cas d'amplification (44), était un biomarqueur prédictif d'une survie globale prolongée pour des GB traités par le protocole STUPP. Par ailleurs, une autre publication de Hobbs et al.(45) montrait que les GB EGFR amplifiés était un groupe hétérogène de GB. Le haut degré d'amplification de EGFR améliorait la réponse au traitement standard.

Des études en TEP aux acides aminés dans les GB démontraient que le volume biologique/métabolique tumoral évalué pourrait également avoir une valeur pronostique. L'étude de Suchorska et al. (46) utilisait le radiopharmaceutique 18Fluoro-Ethyl-Tyrosine (FET). Elle révélait que les $\mathrm{GB}$, avant radiothérapie, avec un volume biologique tumoral faible avaient une meilleure survie. Un article de Jung et al. réalisé avec l'isotope marqué [11C]méthionine (MET) (47) mettait en exergue que les $\mathrm{GB}$, en récidive, avec de petits volumes métaboliques avaient un meilleur pronostic. Dans notre cohorte de patients, il reste à préciser si les différences de volumes biologiques observées selon le statut EGFR sont associées à des différences pronostiques.

En conclusion, notre étude sur les GB récidivants montre que le statut d'amplification génique influence les résultats de la TEP à la 18F-DOPA : les tumeurs présentant " au moins une amplification génique " étaient associées à des intensités de captation plus faibles et les tumeurs EGFR amplifiés étaient corrélés à des volumes biologiques plus petits. Ces données mettent en évidence que l'hétérogénéité moléculaire des GB est associée à une hétérogénéité métabolique.

Ce travail sera complété par une étude de survie pour déterminer si les différences de volumes corrélées au statut EGFR ont une valeur pronostique. 


\section{BIBLIOGRAPHIE}

1. Omuro A, DeAngelis LM. Glioblastoma and Other Malignant Gliomas: A Clinical Review. JAMA. 2013 Nov 6;310(17):1842-50.

2. Louis DN, Perry A, Reifenberger G, Deimling A von, Figarella-Branger D, Cavenee WK, et al. The 2016 World Health Organization Classification of Tumors of the Central Nervous System: a summary. Acta Neuropathol (Berl). 2016 Jun 1;131(6):803-20.

3. Stupp R, Hegi ME, Mason WP, van den Bent MJ, Taphoorn MJB, Janzer RC, et al. Effects of radiotherapy with concomitant and adjuvant temozolomide versus radiotherapy alone on survival in glioblastoma in a randomised phase III study: 5-year analysis of the EORTC-NCIC trial. Lancet Oncol. 2009 May;10(5):459-66.

4. Stupp R, Mason WP, van den Bent MJ, Weller M, Fisher B, Taphoorn MJB, et al. Radiotherapy plus Concomitant and Adjuvant Temozolomide for Glioblastoma. N Engl J Med. 2005 Mar 10;352(10):987-96.

5. Chang JH, Kim C-Y, Choi BS, Kim YJ, Kim JS, Kim IA. Pseudoprogression and Pseudoresponse in the Management of High-Grade Glioma: Optimal Decision Timing According to the Response Assessment of the Neuro-Oncology Working Group. J Korean Neurosurg Soc. 2014 Jan;55(1):5-11.

6. Fink J, Born D, Chamberlain MC. Pseudoprogression: Relevance With Respect to Treatment of High-Grade Gliomas. Curr Treat Options Oncol. 2011 Sep 1;12(3):240.

7. Nabors B, Laterra J. Neuro-Oncology: Current Concepts and Emerging Therapeutics. Neurotherapeutics. 2017 Apr 1;14(2):253-5.

8. Chang PD, Chow DS, Yang PH, Filippi CG, Lignelli A. Predicting Glioblastoma Recurrence by Early Changes in the Apparent Diffusion Coefficient Value and Signal Intensity on FLAIR Images. Am J Roentgenol. 2016 Oct 11;208(1):57-65.

9. Crain ID, Elias PS, Chapple K, Scheck AC, Karis JP, Preul MC. Improving the utility of $1 \mathrm{H}-\mathrm{MRS}$ for the differentiation of glioma recurrence from radiation necrosis. J Neurooncol. 2017 May 1;133(1):97-105.

10. Wan B, Wang S, Tu M, Wu B, Han P, Xu H. The diagnostic performance of perfusion MRI for differentiating glioma recurrence from pseudoprogression. Medicine (Baltimore) [Internet]. 2017 Mar 24 [cited 2017 Aug 5];96(11). Available from: http://www.ncbi.nIm.nih.gov/pmc/articles/PMC5369914/

11. Galldiks N, Langen K-J. Amino acid PET in neuro-oncology: applications in the clinic. Expert Rev Anticancer Ther. 2017 May 4;17(5):395-7.

12. Galldiks N, Law I, Pope WB, Arbizu J, Langen K-J. The use of amino acid PET and conventional MRI for monitoring of brain tumor therapy. Neurolmage Clin. 2017 Jan 1;13:386-94.

13. Walter F, Cloughesy T, Walter MA, Lai A, Nghiemphu P, Wagle N, et al. Impact of 3,4Dihydroxy-6-18F-Fluoro-I-Phenylalanine PET/CT on Managing Patients with Brain Tumors: The Referring Physician's Perspective. J Nucl Med. 2012 Mar 1;53(3):393-8.

14. Darcourt J, Mondot L, Burel-Vandenbos F, Bondiau P-Y, Almairac F, Fontaine D, et al. Clinical impact of 18F-FDOPA PET in the differential diagnosis between recurrence and radiation necrosis in brain tumors. J Nucl Med. 2017 Jan 5;58(supplement 1):75-75.

15. Lizarraga KJ, Allen-Auerbach M, Czernin J, DeSalles AAF, Yong WH, Phelps ME, et al. 18FFDOPA PET for Differentiating Recurrent or Progressive Brain Metastatic Tumors from Late or Delayed Radiation Injury After Radiation Treatment. J Nucl Med. 2014 Jan 1;55(1):30-6.

16. Herrmann K, Czernin J, Cloughesy T, Lai A, Pomykala KL, Benz MR, et al. Comparison of 
visual and semiquantitative analysis of 18F-FDOPA-PET/CT for recurrence detection in glioblastoma patients. Neuro-Oncol. 2014 Apr 1;16(4):603-9.

17. Chen W, Silverman DHS, Delaloye S, Czernin J, Kamdar N, Pope W, et al. 18F-FDOPA PET imaging of brain tumors: comparison study with 18F-FDG PET and evaluation of diagnostic accuracy. J Nucl Med Off Publ Soc Nucl Med. 2006 Jun;47(6):904-11.

18. Dadone-Montaudié B, Ambrosetti D, Dufour M, Darcourt J, Almairac F, Coyne J, et al. [18F] FDOPA standardized uptake values of brain tumors are not exclusively dependent on LAT1 expression. PloS One. 2017;12(9):e0184625.

19. Youland RS, Kitange GJ, Peterson TE, Pafundi DH, Ramiscal JA, Pokorny JL, et al. The role of LAT1 in 18F-DOPA uptake in malignant gliomas. J Neurooncol. 2013 Jan;111(1):11-8.

20. Chiaravalloti A, Fiorentini A, Villani V, Carapella C, Pace A, Di Pietro B, et al. Factors affecting 18F FDOPA standardized uptake value in patients with primary brain tumors after treatment. Nucl Med Biol. 2015 Apr 1;42(4):355-9.

21. Fueger BJ, Czernin J, Cloughesy T, Silverman DH, Geist CL, Walter MA, et al. Correlation of 6-18F-fluoro-L-dopa PET uptake with proliferation and tumor grade in newly diagnosed and recurrent gliomas. J Nucl Med Off Publ Soc Nucl Med. 2010 Oct;51(10):1532-8.

22. Correlation of SUV-Derived Indices With Tumoral... : Clinical Nuclear Medicine [Internet]. LWW.

Available

from: http://journals.Iww.com/nuclearmed/Fulltext/2015/09000/Correlation_of_SUV_Derived_In dices_With_Tumoral.24.aspx

23. FDOPA PET-CT of Nonenhancing Brain Tumors : Clinical Nuclear Medicine [Internet]. LWW. Available from: http://journals.Iww.com/nuclearmed/Fulltext/2017/04000/FDOPA_PET_CT_of_Nonenhanci ng_Brain_Tumors.2.aspx

24. Verger A, Metellus P, Sala Q, Colin C, Bialecki E, Taieb D, et al. IDH mutation is paradoxically associated with higher 18F-FDOPA PET uptake in diffuse grade II and grade III gliomas. Eur J Nucl Med Mol Imaging. 2017 Aug 1;44(8):1306-11.

25. Sala $Q$, Metellus $P$, Chinot $O$, Boyer L, Bialecki E, Dufour $H$, et al. Évaluation préopératoire comparative de 43 gliomes de grade II et III par TEP au 18F-FDG et à la 18F-DOPA : corrélation aux données histologiques et moléculaires. Médecine Nucl. 2016 Aug 1;40(4):285-96.

26. Bosnyák E, Michelhaugh SK, Klinger NV, Kamson DO, Barger GR, Mittal S, et al. Prognostic Molecular and Imaging Biomarkers in Primary Glioblastoma. Clin Nucl Med. 2017 May;42(5):341-7.

27. Liu F, Mischel PS. Targeting epidermal growth factor receptor co-dependent signaling pathways in glioblastoma. Wiley Interdiscip Rev Syst Biol Med. :n/a-n/a.

28. Hatanpaa KJ, Burma S, Zhao D, Habib AA. Epidermal Growth Factor Receptor in Glioma: Signal Transduction, Neuropathology, Imaging, and Radioresistance. Neoplasia N Y N. 2010 Sep;12(9):675-84.

29. Ledezma CJ, Chen W, Sai V, Freitas B, Cloughesy T, Czernin J, et al. 18F-FDOPA PET/MRI fusion in patients with primary/recurrent gliomas: Initial experience. Eur J Radiol. 2009 Aug $1 ; 71(2): 242-8$.

30. Karunanithi S, Sharma P, Kumar A, Khangembam BC, Bandopadhyaya GP, Kumar R, et al. 18F-FDOPA PET/CT for detection of recurrence in patients with glioma: prospective comparison with 18F-FDG PET/CT. Eur J Nucl Med Mol Imaging. 2013 Jul 1;40(7):1025-35.

31. Walter F, Cloughesy T, Walter MA, Lai A, Nghiemphu P, Wagle N, et al. Impact of 3,4Dihydroxy-6-18F-Fluoro-I-Phenylalanine PET/CT on Managing Patients with Brain Tumors: The Referring Physician's Perspective. J Nucl Med. 2012 Mar 1;53(3):393-8. 
32. Kato T, Shinoda J, Nakayama N, Miwa K, Okumura A, Yano H, et al. Metabolic Assessment of Gliomas Using 11C-Methionine, [18F] Fluorodeoxyglucose, and 11C-Choline PositronEmission Tomography. Am J Neuroradiol. 2008 Jun 1;29(6):1176-82.

33. Tsuyuguchi N, Terakawa Y, Uda T, Nakajo K, Kanemura Y. Diagnosis of Brain Tumors Using Amino Acid Transport PET Imaging with 18F-fluciclovine: A Comparative Study with L-methyl11C-methionine PET Imaging. Asia Ocean J Nucl Med Biol. 2017;5(2):85-94.

34. Felsberg J, Hentschel B, Kaulich K, Gramatzki D, Zacher A, Malzkorn B, et al. Prognostic role of Epidermal growth factor receptor variant III (EGFRVIII) positivity in EGFR-amplified primary and recurrent glioblastomas. Clin Cancer Res. 2017 Jan 1;clincanres.0890.2017.

35. Verhaak RGW, Hoadley KA, Purdom E, Wang V, Qi Y, Wilkerson MD, et al. An integrated genomic analysis identifies clinically relevant subtypes of glioblastoma characterized by abnormalities in PDGFRA, IDH1, EGFR and NF1. Cancer Cell. 2010 Jan 19;17(1):98.

36. Nakano I. Stem cell signature in glioblastoma: therapeutic development for a moving target. J Neurosurg. 2014 Nov 14;122(2):324-30.

37. Mao P, Joshi K, Li J, Kim S-H, Li P, Santana-Santos L, et al. Mesenchymal glioma stem cells are maintained by activated glycolytic metabolism involving aldehyde dehydrogenase $1 \mathrm{~A} 3$. Proc Natl Acad Sci. 2013 May 21;110(21):8644-9.

38. Yip SSF, Kim J, Coroller TP, Parmar C, Velazquez ER, Huynh E, et al. Associations Between Somatic Mutations and Metabolic Imaging Phenotypes in Non-Small Cell Lung Cancer. J Nucl Med Off Publ Soc Nucl Med. 2017 Apr;58(4):569-76.

39. Liu A, Han A, Zhu H, Ma L, Huang Y, Li M, et al. The role of metabolic tumor volume (MTV) measured by [18F] FDG PET/CT in predicting EGFR gene mutation status in non-small cell lung cancer. Oncotarget. 2017 Apr 4;8(20):33736-44.

40. Takamochi K, Mogushi K, Kawaji H, Imashimizu K, Fukui M, Oh S, et al. Correlation of EGFR or KRAS mutation status with 18F-FDG uptake on PET-CT scan in lung adenocarcinoma. PLOS ONE. 2017 Apr 19;12(4):e0175622.

41. Biau J, Dalloz P, Durando X, Hager M-O, Ouédraogo ZG, Khalil T, et al. Glioblastome du sujet âgé : état de l'art. Bull Cancer (Paris). 2015 Mar 1;102(3):277-86.

42. Chen J-R, Xu H-Z, Yao Y, Qin Z-Y. Prognostic value of epidermal growth factor receptor amplification and EGFRvIll in glioblastoma: meta-analysis. Acta Neurol Scand. 2015 Nov 1;132(5):310-22.

43. Montano N, Cenci T, Martini M, D’Alessandris QG, Pelacchi F, Ricci-Vitiani L, et al. Expression of EGFRvIII in Glioblastoma: Prognostic Significance Revisited. Neoplasia N Y N. 2011 Dec;13(12):1113-21.

44. Weller M, van den Bent M, Hopkins K, Tonn JC, Stupp R, Falini A, et al. EANO guideline for the diagnosis and treatment of anaplastic gliomas and glioblastoma. Lancet Oncol. 2014 Aug;15(9):e395-403.

45. Hobbs J, Nikiforova MN, Fardo DW, Bortoluzzi S, Cieply K, Hamilton RL, et al. Paradoxical relationship between degree of EGFR amplification and outcome in glioblastomas. Am J Surg Pathol. 2012 Aug;36(8):1186-93.

46. Suchorska B, Jansen NL, Linn J, Kretzschmar H, Janssen H, Eigenbrod S, et al. Biological tumor volume in 18FET-PET before radiochemotherapy correlates with survival in GBM. Neurology. 2015 Feb 17;84(7):710-9.

47. Jung T-Y, Min J-J, Bom H-S, Jung S, Kim I-Y, Lim S-H, et al. Prognostic value of posttreatment metabolic tumor volume from 11C-methionine PET/CT in recurrent malignant glioma. Neurosurg Rev. 2017 Apr 1;40(2):223-9. 


\section{ANNEXES}

Annexe 1. Classification histo-pronostique OMS 2016 des glioblastomes.

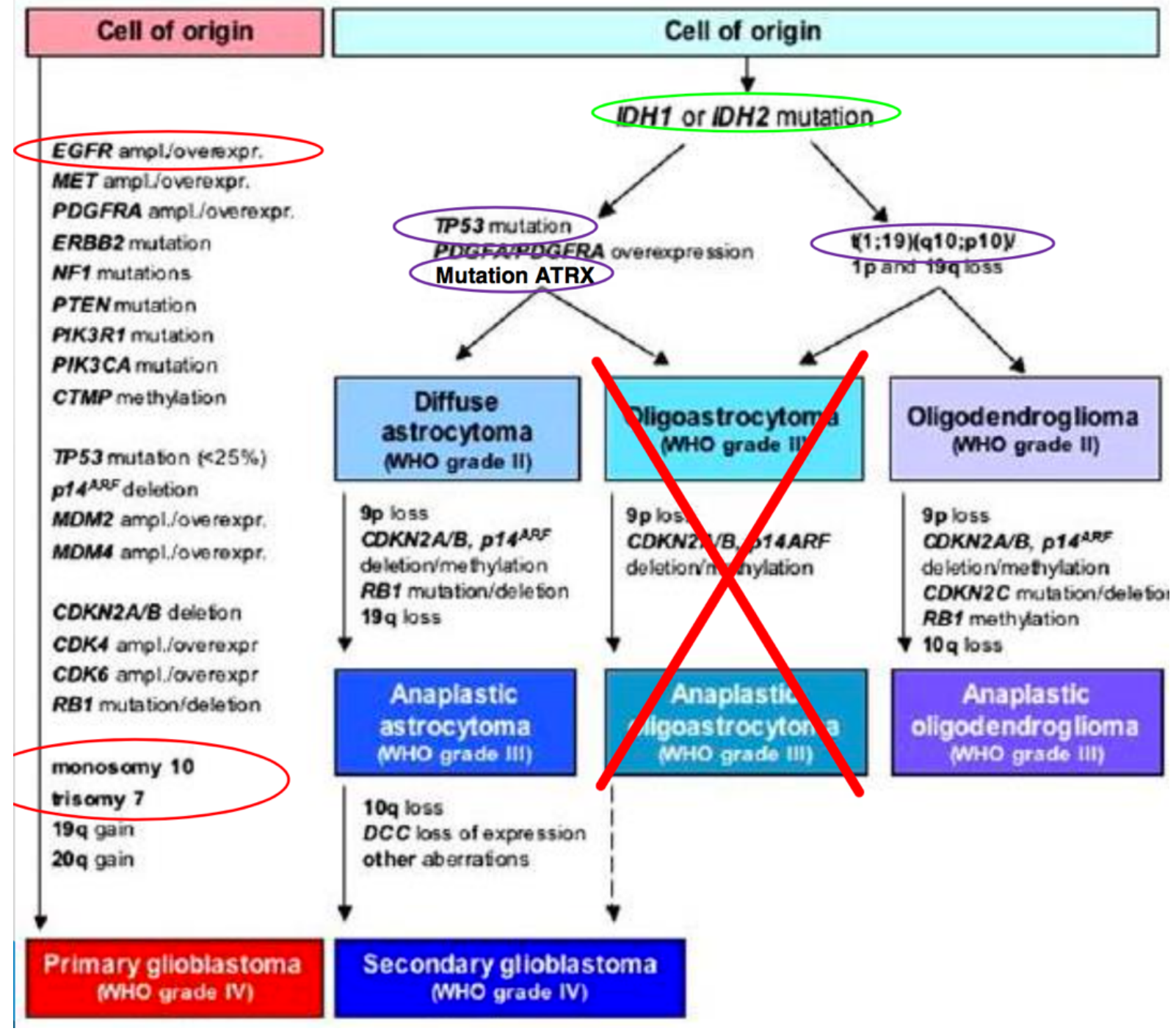


Annexe 2. Echelle visuelle selon Lizarraga pour différencier récidive et radionécrose.

0 : lésion non visible

1 : lésion captant moins que le striatum controlatéral

2 : lésion captant autant que le striatum controlatéral

3 : lésion captant plus que le striatum controlatéral
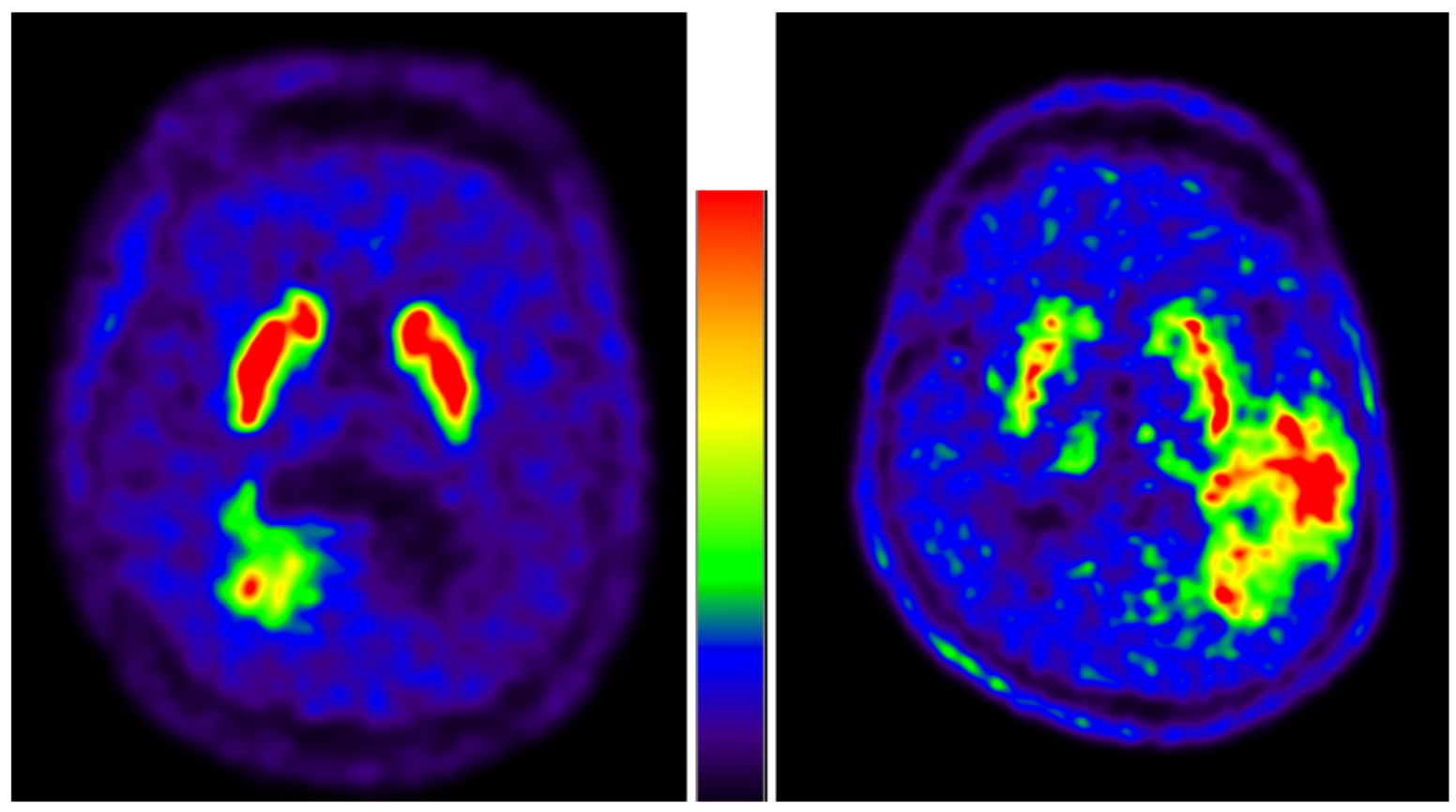

Score 2

Score 3 
Annexe 3. Voies de signalisation affectées par les amplifications géniques dans les GB.
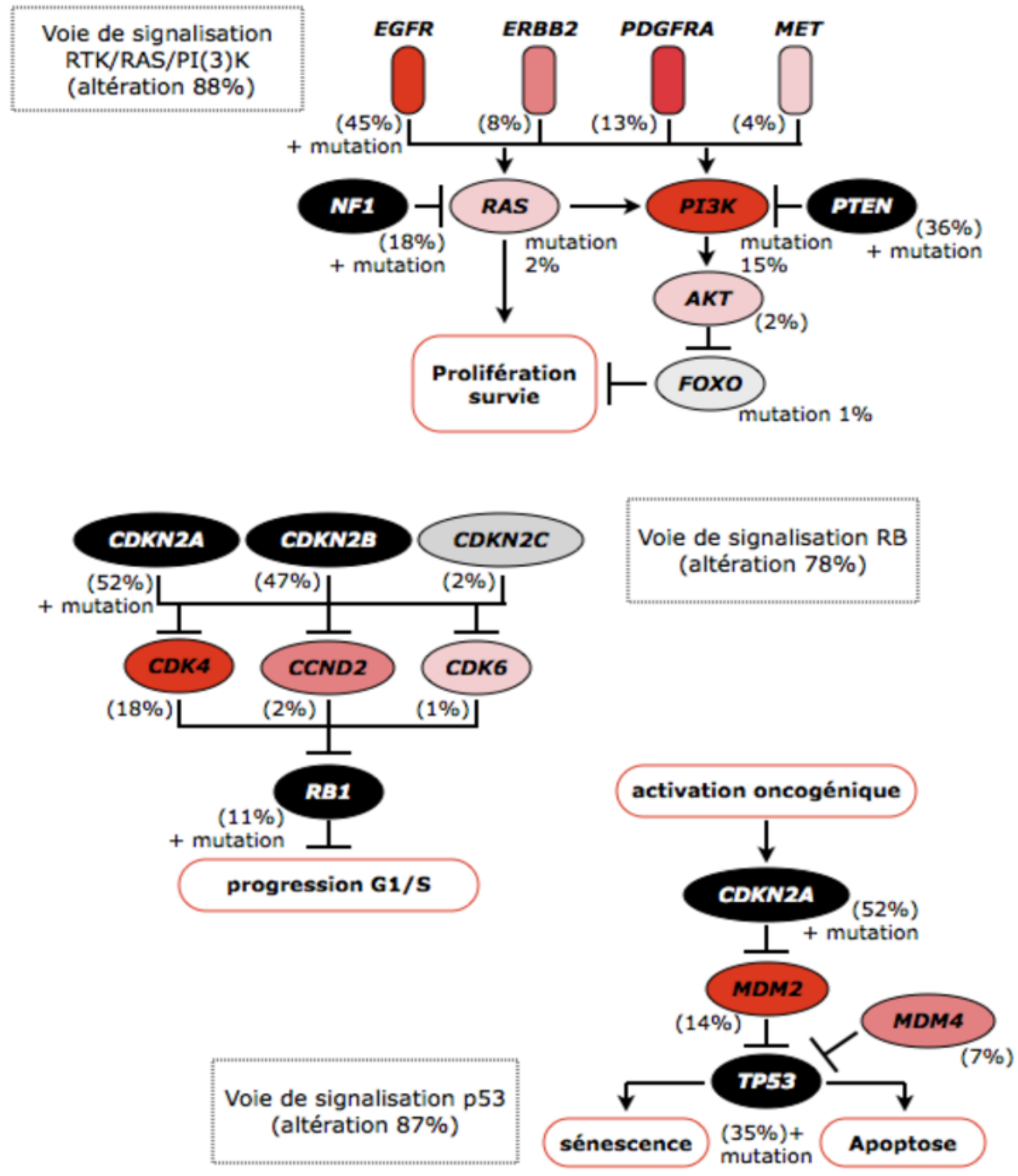
Annexe 4. Résumé des critères RANO publiés en 2010

\begin{tabular}{|l|c|c|c|c|}
\hline Critères & $\begin{array}{c}\text { Réponse } \\
\text { complète }\end{array}$ & $\begin{array}{c}\text { Réponse } \\
\text { partielle }\end{array}$ & Maladie stable & Progression \\
\hline $\begin{array}{l}\text { Prise de } \\
\text { gadolinium }\end{array}$ & Absence & $\begin{array}{c}\text { Diminution } \\
\text { supérieure ou } \\
\text { égale } 50 \%\end{array}$ & $\begin{array}{c}\text { Régression de } \\
\text { moins de } 50 \% \\
\text { ou progression } \\
\text { de moins de } \\
25 \%\end{array}$ & $\begin{array}{c}\text { Augmention de } \\
\text { plus de } 25 \%[1]\end{array}$ \\
\hline T2/Flair & Stable ou diminué & $\begin{array}{c}\text { Stable ou } \\
\text { diminué }\end{array}$ & $\begin{array}{c}\text { Stable ou } \\
\text { diminué }\end{array}$ & Augmentation\% ${ }^{[1]}$ \\
\hline $\begin{array}{l}\text { Nouvelle(s) } \\
\text { lésion(s) }\end{array}$ & Absence & Absence & Absence & Absence \\
\hline Stéroïdes & Absence & $\begin{array}{c}\text { Stable ou } \\
\text { diminué }\end{array}$ & $\begin{array}{c}\text { Stable ou } \\
\text { diminué }\end{array}$ & Non applicable ${ }^{[2]}$ \\
\hline Statut clinique & $\begin{array}{c}\text { Stable ou } \\
\text { amélioré }\end{array}$ & $\begin{array}{c}\text { Stable ou } \\
\text { amélioré }\end{array}$ & $\begin{array}{c}\text { Stable ou } \\
\text { amélioré }\end{array}$ & Altéré ${ }^{[1]}$ \\
\hline $\begin{array}{l}\text { Nécessité pour } \\
\text { réponse }\end{array}$ & Tous & Tous & Tous & Tous ${ }^{[1]}$ \\
\hline
\end{tabular}


Annexe 5. Sous-types moléculaires de GB selon la classification de Verhaak (2010)

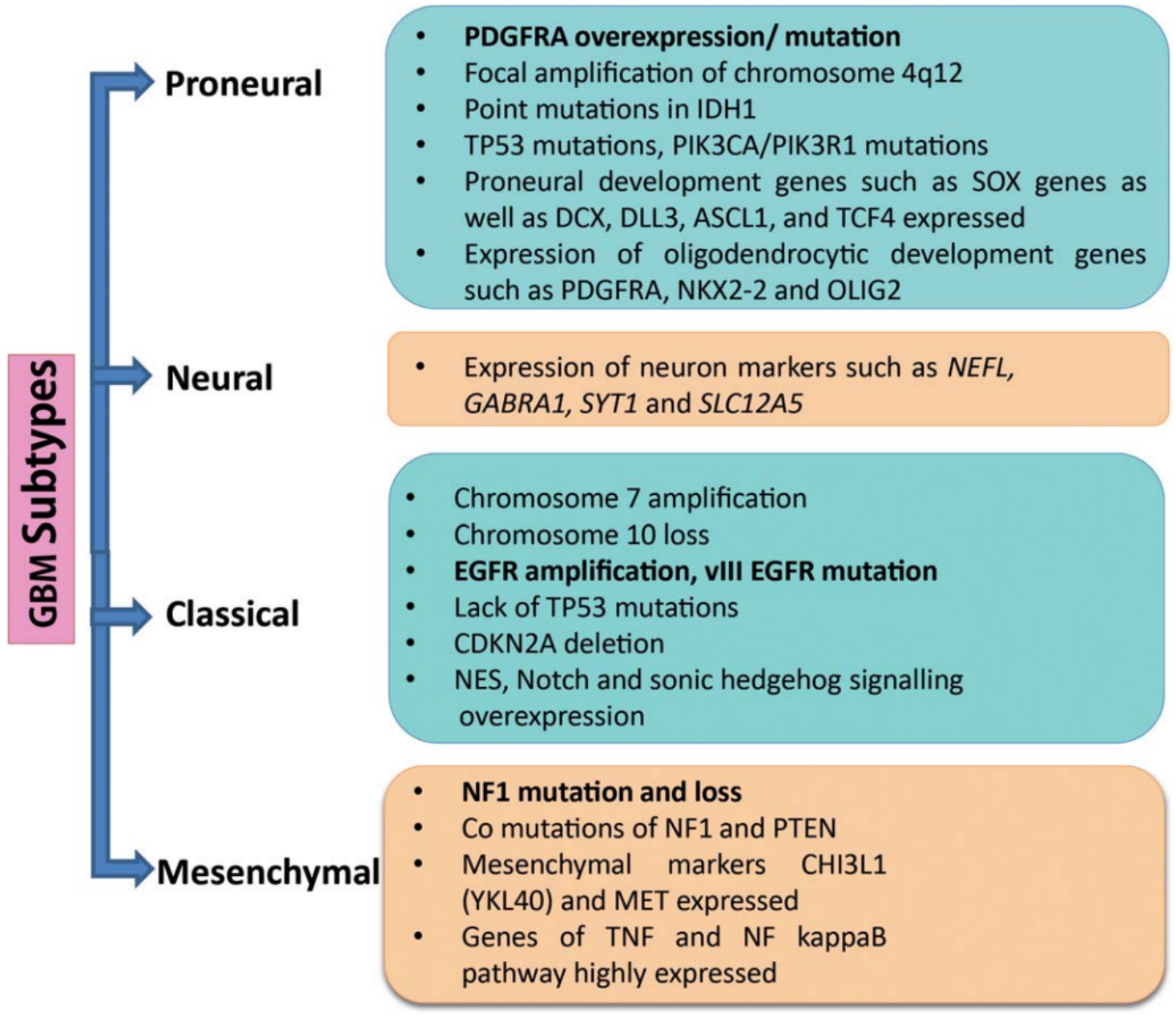




\section{ABREVIATIONS}

\section{GB : glioblastome}

OMS : Organisation Mondiale de la Santé

$\mathrm{IDH}$ : isocitrate déshydrogénase

$\mathrm{RCT}$ : radio-chimiothérapie

IRM : imagerie par résonnance magnétique

TEP : tomoscintigraphie par émission de positons

18F-DOPA: 6-18F-fluoro-L-3,4-dihydroxy-phenylalanine

$\mathrm{RCP}$ : réunion de concertation pluridisciplinaire

LAT1 : transporteur d'acides aminés L-type amino-acid transporter 1

mTOR : cible de la ripamycine chez les mammifères

$\mathrm{RT}$ : radiothérapie

Ki-67 : index d'activité mitotique

MGMT: 06-methylguanine-DNA methyl-transferase

EGFR: receptor epidermal growth factor

MET: mesenchymal-epithelial transition

PDGFRA: alpha platelet-derived growth factor

CDK4 : cyclin-dependent kinase 4

MDM2 : murine double minute 2

MDM4 : murine double minute 4

AMT : alpha[C-11] -L-methyl-tryptophane

ADC : coefficient apparent de diffusion

$\mathrm{T} / \mathrm{S}$ : tumeur/striatum controlatéral

SUV : Standard Uptake Value

SUV max : SUV maximale dans un pixel

SUV peak : SUV moyen dans une sphère de $1 \mathrm{~cm}^{3}$ centrée sur le maximum

$\mathrm{T} / \mathrm{N}$ : tumeur/hémisphère sain

SUV mean $\mathrm{N}$ : SUV moyen de l'hémisphère sain controlatéral

SUV mean S : SUV moyen du striatum

Vol $1.6 \mathrm{~N}$ : volume $1.6 \mathrm{~N}$

FET : 18Fluoro-Ethyl-Tyrosine

$\mathrm{IHC}$ : immunohisto-chimie

CGH : Comparative Génomic Hybridization

Vol $0.8 \mathrm{~S}$ et Vol0.6S : les 2 volumes biologiques tumoraux, calculés par rapport au striatum.

NGS: Next Generation Sequencing

CGH: Comparative genomic hybridization

VOI : volume d'intérêt

C-11 MET : C-11 Méthionine 


\section{SERMENT D'HIPPOCRATE}

Au moment d'être admis à exercer la médecine, je promets et je jure d'être fidèle aux lois de l'honneur et de la probité.

Mon premier souci sera de rétablir, de préserver ou de promouvoir la santé dans tous ses éléments, physiques et mentaux, individuels et sociaux.

Je respecterai toutes les personnes, leur autonomie et leur volonté, sans aucune discrimination selon leur état ou leurs convictions. J'interviendrai pour les protéger si elles sont affaiblies, vulnérables ou menacées dans leur intégrité ou leur dignité. Même sous la contrainte, je ne ferai pas usage de mes connaissances contre les lois de l'humanité.

J'informerai les patients des décisions envisagées, de leurs raisons et de leurs conséquences. Je ne tromperai jamais leur confiance et n'exploiterai pas le pouvoir hérité des circonstances pour forcer les consciences.

Je donnerai mes soins à l'indigent et à quiconque me les demandera. Je ne me laisserai pas influencer par la soif du gain ou la recherche de la gloire.

Admis dans l'intimité des personnes, je tairai les secrets qui me seront confiés. Reçu à l'intérieur des maisons, je respecterai les secrets des foyers et ma conduite ne servira pas à corrompre les mœurs.

Je ferai tout pour soulager les souffrances. Je ne prolongerai pas abusivement les agonies. Je ne provoquerai jamais la mort délibérément.

Je préserverai l'indépendance nécessaire à l'accomplissement de ma mission. Je n'entreprendrai rien qui dépasse mes compétences. Je les entretiendrai et les perfectionnerai pour assurer au mieux les services qui me seront demandés.

J'apporterai mon aide à mes confrères ainsi qu'à leurs familles dans l'adversité.

Que les hommes et mes confrères m'accordent leur estime si je suis fidèle à mes promesses ; que je sois déshonoré et méprisé si j’y manque. 


\section{RÉSUMÉ}

Introduction. Les mécanismes d'accumulation de la 18F-DOPA en Tomographie par Emission de Positons (TEP) dans les glioblastomes (GB) restent mal connus.

L'objectif de ce travail était de rechercher si les données de la TEP à la 18F-DOPA en situation de récidive pouvaient être influencées par les amplifications géniques présentes dans les $G B$. Méthodes. Trente et un patients présentant une récidive avec un GB primaire (gène IDH sauvage) ont été inclus rétrospectivement ( 25 résections et 6 biopsies). Les paramètres de la TEP à la 18F-DOPA étaient les intensités et les volumes tumoraux par rapport au striatum ou par rapport à l'hémisphère sain : $\mathrm{T} / \mathrm{S}, \mathrm{T} / \mathrm{N}, \mathrm{Vol}_{(\mathrm{S})}$ et $\mathrm{Vol}_{(\mathrm{N})}$. Les données moléculaires ont été classées en 2 groupes selon la présence ou l'absence d'une amplification génique.

Résultats. Les intensités de captation de la 18F-DOPA étaient inversement corrélées au statut d'amplification génique $(p=0,031)$. Les volumes biologiques tumoraux étaient supérieurs pour les GB EGFR non amplifiés (Vol $\mathrm{l}_{0.6 \mathrm{~s}}$ médian à $3 \mathrm{~cm}^{3}$ contre $14 \mathrm{~cm}^{3}$ pour les GB EGFR amplifiés ; $p=0,029$ ) dans la population totale et dans le sous-groupe des résections chirurgicales.

Conclusion. Les amplifications géniques étaient associées à des modifications de captation de la 18F-DOPA lors de la récidive tumorale, comparativement aux GB sans amplification : les GB présentant au moins une amplification génique étaient associés à des intensités de captation plus faibles et les GB EGFR amplifiés étaient corrélées à des volumes biologiques tumoraux plus petits.

Mots clés : glioblastome, IDH sauvage, récidive, TEP, 18F-DOPA, amplification génique, EGFR. 